\title{
Uncertainty quantification in operational modal analysis with stochastic subspace identification: validation and applications
}

\author{
Edwin Reynders*, Kristof Maes, Geert Lombaert and Guido De Roeck \\ University of Leuven (KU Leuven), Department of Civil Engineering, Kasteelpark Arenberg 40, B-3001 Leuven, Belgium
}

\begin{abstract}
Identified modal characteristics are often used as a basis for the calibration and validation of dynamic structural models, for structural control, for structural health monitoring, etc. It is therefore important to know their accuracy. In this article, a method for estimating the (co)variance of modal characteristics that are identified with the stochastic subspace identification method is validated for two civil engineering structures. The first structure is a damaged prestressed concrete bridge for which acceleration and dynamic strain data were measured in 36 different setups. The second structure is a mid-rise building for which acceleration data were measured in 10 different setups. There is a good quantitative agreement between the predicted levels of uncertainty and the observed variability of the eigenfrequencies and damping ratios between the different setups. The method can therefore be used with confidence for quantifying the uncertainty of the identified modal characteristics, also when some or all of them are estimated from a single batch of vibration data. Furthermore, the method is seen to yield valuable insight in the variability of the estimation accuracy from mode to mode and from setup to setup: the more informative a setup is regarding an estimated modal characteristic, the smaller is the estimated variance.
\end{abstract}

Keywords: operational modal analysis, stochastic subspace identification, variance estimation

\section{Introduction}

The problem of quantifying the uncertainty of modal characteristics that are estimated from a single batch of (operational) vibration data gained interest during the past years, with the development of expressions for the variance of the estimates obtained from two high-performance system identification methods: stochastic subspace identification [1] and maximum likelihood estimation [2]. These expressions enable the estimation of the variance of the eigenfrequencies, damping ratios, and mode shapes, obtained from a single batch of data, as well as the covariance between them. They also allow computing confidence intervals because the estimates of the modal characteristics are asymptotically normally distributed.

In this article, the variance estimation procedure is validated for the stochastic subspace identification (SSI) method, which has become a standard for operational modal analysis $[3,4,5]$. SSI essentially estimates a state-space model from an observed output correlation sequence using linear algebra techniques. An eigenvalue decomposition of the identified state-space model then yields the eigenfrequencies, damping ratios and mode shapes. Several algorithmic variants of SSI exist, amongst which SSI-cov (covariance-driven stochastic subspace identification) [6, 7] and NExT-ERA (eigensystem realization algorithm combined with the natural excitation technique) $[8,9,10]$ are very commonly employed in operational modal analysis. These algorithms are all specific implementations of a single basic algorithm [11] and their computational and statistical performance is largely similar in practice $[3,5]$.

The SSI method has the particular advantage of combining a high estimation accuracy with a high computational robustness and efficiency. Optimality of the estimation accuracy in the sense of asymptotic minimum variance (also called statistical efficiency) has also been theoretically proven for some implementations of SSI [12]. The accuracy of SSI estimation is therefore comparable to that of maximum likelihood (ML) estimation, for which asymptotic statistical

\footnotetext{
*Corresponding author: Tel.: +32 16321677; fax: +32 16321988; E-mail address: edwin.reynders@ bwk.kuleuven.be. Postprint submitted to Mechanical Systems \& Signal Processing

Published version: E. Reynders, K. Maes, G.Lombaert, and G. De Roeck. Uncertainty quantification in operational modal analysis with stochastic subspace identification: validation and applications. Mechanical Systems \& Signal Processing, 66-67:13-30, 2016. http://dx.doi.org/10.1016/j.ymssp.2015.04.018
} 
efficiency is guaranteed $[13,14,15]$. However, ML estimation is computationally costly because it involves the iterative minimization of a non-convex objective function. Furthermore, simulations have shown that the method can get stuck in local minima even for starting values that are very close to the exact values [5]. In order to reduce the computation cost, an approximate ML estimation is usually performed in which the covariance between the different output estimates is disregarded [16]. Note that the Bayesian modal identification algorithms that have been recently proposed essentially reduce to maximum likelihood estimation since prior information is not employed [17]. Expressions for the variance of the estimated modal characteristics also exist for the p-LSCF method (poly-reference least squares complex frequency domain) [18]. This method results in clear stabilization diagrams which facilitate the modal identification process, but it can also result in biased estimates, especially of the damping ratio [5], and therefore the variance alone may largely underestimate the total statistical uncertainty of the modal characteristics that are obtained with this method.

The covariance estimation procedure for the modal characteristics (or state-space model parameters) estimated with SSI is based on a first-order sensitivity analysis of the identified values to the output correlations where the algorithm starts from. This sensitivity analysis, the related covariance expressions, and a numerical verification have been presented in [1]. An implementation of the covariance expressions that is optimized in terms of computational efficiency was presented in [19], and an extension towards the joint analysis of multi-setup measurements was presented in [20].

In the present article, the method for estimating the (co)variance of modal characteristics that are identified with SSI is validated for two civil engineering structures: a damaged prestressed concrete bridge for which acceleration and dynamic strain data were measured in 36 different setups, and a mid-rise building for which acceleration data were measured in 10 different setups. For each structure, the validation consists of two stages. In the first stage, the estimated uncertainty of the identified eigenfrequencies and damping ratios is compared with the observed variability across all setups. The observed variance (or sample variance) and the predicted variance should be the same if the amount of information contained in the data is the same in all setups and the test conditions are the same. This can reasonably be expected when the measurements for all setups are carried out in similar circumstances. The second stage focuses on the differences in estimation accuracy between different modes and different setups: if a particular experiment is less informative on a first mode than it is on a second mode, e.g. because the first mode is less well excited, then the predicted uncertainty should be larger for the first mode than for the second mode.

The remainder of this article is organized as follows. In section 2, the estimation of modal characteristics with SSI and the computation of the variance of these estimates are briefly summarized. Section 3 contains the first detailed validation study, which involves the ambient modal testing of a damaged prestressed concrete bridge. The second validation study, comprising the identification of the modal characteristics of a mid-rise building under ambient wind excitation, is discussed in section 4 . The final conclusions are presented in section 5.

\section{Stochastic subspace identification with uncertainty quantification}

As indicated previously, several algorithmic variants of SSI exist. The following analysis concentrates on the SSI-cov algorithm because of two reasons: the algorithm is simple and easy to implement, and it is also theoretically optimal in the sense that the resulting system description is deterministically balanced [21]. The SSI-cov algorithm is also known under the name principal component (PC) algorithm [11].

\subsection{System model}

The input-output relationship of a linear time-invariant system can, after discretization in time, be described with the following state-space model:

$$
\begin{aligned}
\mathbf{x}_{k+1} & =\mathbf{A} \mathbf{x}_{k}+\mathbf{B} \mathbf{u}_{k} \\
\mathbf{y}_{k} & =\mathbf{C} \mathbf{x}_{k}+\mathbf{D} \mathbf{u}_{k}+\mathbf{e}_{k}
\end{aligned}
$$

where $\mathbf{x}_{k} \in \mathbb{R}^{n}$ is the state of the system, $\mathbf{y}_{k} \in \mathbb{R}^{n_{\mathrm{y}}}$ is the output vector, $\mathbf{u}_{k} \in \mathbb{R}^{n_{\mathrm{u}}}$ is the input vector, and $\mathbf{A}, \mathbf{B}, \mathbf{C}$ and $\mathbf{D}$ are the system matrices. In operational modal analysis, only the outputs $\mathbf{y}_{k}$ are observed, with measurement error $\mathbf{e}_{k}$. In general, little information on the loading $\mathbf{u}_{k}$ and the measurement error $\mathbf{e}_{k}$ is available, other than that they take finite values that are centered around zero. For this reason $\mathbf{u}_{k}$ and $\mathbf{e}_{k}$ are modeled as white noise random processes. This is a good assumption as long as the ambient excitation spectrum is not dominated by specific frequency components, which is for instance the case when strong harmonics are present in the excitation [5]. The white noise model also follows naturally 
from a Bayesian perspective, because it represents a state of maximum information entropy (or maximum uncertainty) given the available information [22].

Since $\mathbf{u}_{k}$ and $\mathbf{e}_{k}$ are assumed to be discrete white noise random processes, the state-space model can be reformulated as

$$
\begin{aligned}
\mathbf{x}_{k+1} & =\mathbf{A} \mathbf{x}_{k}+\mathbf{w}_{k} \\
\mathbf{y}_{k} & =\mathbf{C x}_{k}+\mathbf{v}_{k},
\end{aligned}
$$

where $\mathbf{w}_{k}:=\mathbf{B} \mathbf{u}_{k}$ and $\mathbf{v}_{k}:=\mathbf{D} \mathbf{u}_{k}+\mathbf{e}_{k}$ are called the process noise and output noise, respectively, and

$$
\mathrm{E}\left(\left[\begin{array}{l}
\mathbf{w}_{k} \\
\mathbf{v}_{k}
\end{array}\right]\left[\begin{array}{l}
\mathbf{w}_{k+l} \\
\mathbf{v}_{k+l}
\end{array}\right]^{\mathrm{T}}\right)=\left[\begin{array}{cc}
\mathbf{Q} & \mathbf{S} \\
\mathbf{S}^{\mathrm{T}} & \mathbf{R}
\end{array}\right] \delta_{1}(l) .
$$

The covariance matrices $\mathbf{Q} \in \mathbb{R}^{n \times n}, \mathbf{S} \in \mathbb{R}^{n \times n_{\mathrm{y}}}$ and $\mathbf{R} \in \mathbb{R}^{n_{\mathrm{y}} \times n_{\mathrm{y}}}$ are time-invariant, and $\delta_{1}(l)$ represents the unit impulse function, i.e., $\delta_{1}(0)=1$ and $\delta_{1}(l)=0, l \neq 0$.

The prime goal in operational modal analysis is to identify the system matrices $\mathbf{A}$ and $\mathbf{C}$ from the observed output sequence $\mathbf{y}_{k}$, because the knowledge of $\mathbf{A}$ and $\mathbf{C}$ is sufficient for estimating the modal characteristics. Indeed, an eigenvalue decomposition of the matrix $\mathbf{A}$,

$$
\mathbf{A}=\sum_{k=1}^{n} \boldsymbol{\psi}_{k} \lambda_{k} \boldsymbol{\psi}_{k}^{\mathrm{T}}
$$

yields the continuous-time eigenvalues $\lambda_{\mathbf{c} k}$, eigenfrequencies $f_{k}$, damping ratios $\xi_{k}$ (in $\%$ of critical) [5]:

$$
\lambda_{\mathrm{c} k}=\frac{\ln \lambda_{k}}{T}, \quad f_{k}=\frac{\left|\lambda_{\mathbf{c} k}\right|}{2 \pi}, \quad \text { and } \quad \xi_{k}=-100 \frac{\mathcal{R}\left(\lambda_{\mathbf{c} k}\right)}{\left|\lambda_{\mathbf{c} k}\right|},
$$

where $\mathcal{R}$ ( ) denotes the real part of a variable, and $T$ denotes the sampling period. Combining the eigenvectors of $\mathbf{A}$ with $\mathrm{C}$ yields the mode shapes $\phi_{k}$ in terms of the measured quantities:

$$
\phi_{k}=\mathbf{C} \psi_{k}
$$

\subsection{System matrices and modal characteristics: point estimates}

From the previous discussion, it is clear that, since $\mathbf{w}_{k}$ and $\mathbf{v}_{k}$ are not precisely known, the observed output sequence $\mathbf{y}_{k}$ is a random process. In order to obtain the system matrices, the output correlation matrices $\boldsymbol{\Lambda}_{l} \in \mathbb{R}^{n_{\mathrm{y}} \times n_{\mathrm{ref}}}$, which are defined as

$$
\boldsymbol{\Lambda}_{l}:=\mathrm{E}\left(\mathbf{y}_{k+l} \mathbf{y}_{k}^{\mathrm{ref}}\right)
$$

where $\mathrm{E}$ denotes the expectation operator, are estimated for different time lags: $l=1, \ldots, 2 i-1$. In this expression, $\mathbf{y}_{k}^{\text {ref }} \in \mathbb{R}^{n_{\text {ref }}}$ stands for the vector with reference outputs. The set of reference outputs is a subset of the set of measured outputs, which is mainly employed to increase the computational efficiency of the algorithm when many outputs are measured. When the modal test is performed in different setups, the outputs that are common to all setups are usually good candidates as reference outputs in the algorithm [7]. The following relation between the output correlation matrices and the system matrices follows directly from (3-5):

$$
\boldsymbol{\Lambda}_{l}=\mathbf{C A}^{l-1} \mathbf{G}, \quad l>0,
$$

where

$$
\mathbf{G}:=\mathrm{E}\left[\mathbf{x}_{k+1} \mathbf{y}_{k}^{\mathrm{ref}}\right]
$$

The SSI-cov algorithm starts by collecting the output correlation matrices in a large Hankel matrix $\mathbf{L}_{1 \mid i} \in \mathbb{R}^{i n_{\mathrm{y}} \times i n_{\mathrm{ref}}}$ :

$$
\mathbf{L}_{1 \mid i}:=\left[\begin{array}{cccc}
\boldsymbol{\Lambda}_{1} & \boldsymbol{\Lambda}_{2} & \ldots & \boldsymbol{\Lambda}_{i} \\
\boldsymbol{\Lambda}_{2} & \boldsymbol{\Lambda}_{3} & \ldots & \boldsymbol{\Lambda}_{i+1} \\
\ldots & \ldots & \ldots & \ldots \\
\boldsymbol{\Lambda}_{i} & \boldsymbol{\Lambda}_{i+1} & \ldots & \boldsymbol{\Lambda}_{2 i-1}
\end{array}\right]
$$


With the identity (10), this matrix decomposes as

$$
\mathbf{L}_{1 \mid i}:=\underbrace{\left[\begin{array}{c}
\mathbf{C} \\
\mathbf{C A} \\
\ldots \\
\mathbf{C A}^{i-1}
\end{array}\right]}_{:=\mathcal{O}_{i}} \underbrace{\left[\begin{array}{llll}
\mathbf{G} & \mathbf{A G} & \ldots & \mathbf{A}^{i-1} \mathbf{G}
\end{array}\right]}_{\mathcal{C}_{i}^{\mathrm{S}}} .
$$

The matrices $\mathcal{O}_{i}$ and $\mathcal{C}_{i}^{\mathrm{S}}$ are called the extended observability and stochastic controllability matrices, respectively. They can be obtained from $\mathbf{L}_{1 \mid i}$, up to a similarity transformation of the matrix $\mathbf{A}$, using reduced singular value decomposition $[23,24]$ :

$$
\mathbf{L}_{1 \mid i}=\mathbf{U} \boldsymbol{\Sigma} \mathbf{V}^{\mathrm{T}}, \quad \mathcal{O}_{i}=\mathbf{U} \boldsymbol{\Sigma}^{1 / 2}, \quad \mathcal{C}_{i}^{\mathrm{S}}=\boldsymbol{\Sigma}^{1 / 2} \mathbf{V}^{\mathrm{T}}
$$

where $\boldsymbol{\Sigma} \in \mathbb{R}^{n \times n}$ contains only the nonzero singular values and $\mathbf{U} \in \mathbb{R}^{i n_{y} \times n}$ and $\mathbf{V} \in \mathbb{R}^{i n_{\text {ref }} \times n}$ the corresponding singular vectors.

The matrices $\mathbf{A}$ and $\mathbf{C}$ are easily retrieved from the extended observability matrix $\mathcal{O}_{i}$. The matrix $\mathbf{C}$ can be determined as the first block of $n_{y}$ rows of $\mathcal{O}_{i}$. The matrix $\mathbf{A}$ follows from the shift structure of $\mathcal{O}_{i}[24]$ :

$$
\mathbf{A}=\underline{\mathcal{O}}_{i}^{\dagger} \overline{\mathcal{O}_{i}},
$$

where $\underline{\mathcal{O}_{i}}$ is equal to $\mathcal{O}_{i}$ without the last $n_{y}$ rows and $\overline{\mathcal{O}_{i}}$ is equal to $\mathcal{O}_{i}$ without the first $n_{y}$ rows. Once the system matrices $\mathbf{A}$ and $\mathbf{C}$ are known, the modal characteristics follow immediately from (7-8).

It may be noted that the model order $n$ has been assumed known up to this point. The model order determines the number of modes: a structure with model order $n$ has $n / 2$ modes [5]. In modal testing, it is therefore customary to estimate the system matrices $\mathbf{A}$ and $\mathbf{C}$ for a wide range of model orders, and to compute the corresponding modal characteristics. Modes that appear at many orders are then called stable modes, and for each stable mode representative characteristics are determined at a specific model order in a stabilization diagram [25].

Finally, the parameter $i$ that determines the size of $\mathbf{L}_{1 \mid i}$, should also be specified by the user. In theory, any value of $i$ that is larger than ceil $\left(n_{\max } / n_{\mathrm{y}}\right)+1$, with $n_{\max }$ the maximal model order that is needed for constructing the stabilization diagram, can be adopted. In practice, larger values of $i$ often yield more accurate system estimates, because they add redundancy to the estimation procedure. A large value of $i$ is also needed when the ratio between the sampling frequency and the fundamental eigenfrequency of the system is large, as detailed in [26, Sec. 3.3]. However, very large values of $i$ result in a considerable computation cost and memory usage, so a trade-off is necessary. The values that are employed in this paper reflect such a trade-off. It has been verified that the reported results are not very sensitive to the particular value of $i$ that is adopted.

\subsection{System matrices and modal characteristics: variance of the estimates}

The output correlation matrices, where the SSI algorithm starts from, are not precisely known in practice: they are estimated by computing sample correlations from the available output data:

$$
\hat{\boldsymbol{\Lambda}}_{l}:=\frac{1}{j} \sum_{k=1}^{j} \mathbf{y}_{k+l} \mathbf{y}_{k}^{\mathrm{ref}}
$$

where the number of available samples $j$ equals the total number of samples minus twice the number of block rows $i$ in the matrix $\mathbf{L}_{1 \mid i}$. As a result, the estimated system matrices $\mathbf{A}$ and $\mathbf{C}$ and the estimated eigenfrequencies $f_{k}$, damping ratios $\xi_{k}$ and mode shapes $\phi_{k}$ that result from the SSI-cov algorithm, are not exact. In order to compute the full covariance between all modal characteristics, a first-order sensitivity analysis of the SSI-cov algorithm can be performed. This yields, for each model order $n$, the Jacobian $\mathbf{J}_{\mathrm{AC}, \Lambda}$ that relates small changes in the elements of the sample correlation matrices $\Delta \boldsymbol{\Lambda}_{l}$ to changes in the estimates of the elements of the system matrices ( $\Delta \mathbf{A}$ and $\Delta \mathbf{C})$ [1]:

$$
\left[\begin{array}{c}
\operatorname{vec}(\Delta \mathbf{A}) \\
\operatorname{vec}(\Delta \mathbf{C})
\end{array}\right]=\mathbf{J}_{\mathrm{AC}, \Lambda} \operatorname{vec}\left(\Delta \boldsymbol{\Lambda}_{1 \mid 2 i-1}\right),
$$


where vec () denotes the operator that stacks the columns of a matrix on top of each other, and

$$
\boldsymbol{\Lambda}_{1 \mid 2 i-1}:=\left[\begin{array}{c}
\boldsymbol{\Lambda}_{1} \\
\boldsymbol{\Lambda}_{2} \\
\vdots \\
\boldsymbol{\Lambda}_{2 i-1}
\end{array}\right] .
$$

Let us denote the covariance matrix between any two real random vectors $\hat{\mathbf{x}}$ and $\hat{\mathbf{y}}$ by

$$
\operatorname{cov}(\hat{\mathbf{x}}, \hat{\mathbf{y}}):=\mathrm{E}\left((\hat{\mathbf{x}}-\mathrm{E}(\hat{\mathbf{x}}))(\hat{\mathbf{y}}-\mathrm{E}(\hat{\mathbf{y}}))^{\mathrm{T}}\right)
$$

Suppose for a moment that the full covariance matrix between all elements of $\hat{\Lambda}_{1 \mid 2 i-1}$ is known. The full covariance matrix between the elements of the system matrices can then be obtained, up to first order, from

$$
\operatorname{cov}\left(\left[\begin{array}{c}
\operatorname{vec}\left(\hat{\mathbf{A}}_{n_{1}}\right) \\
\operatorname{vec}\left(\hat{\mathbf{C}}_{n_{1}}\right)
\end{array}\right],\left[\begin{array}{l}
\operatorname{vec}\left(\hat{\mathbf{A}}_{n_{2}}\right) \\
\operatorname{vec}\left(\hat{\mathbf{C}}_{n_{2}}\right)
\end{array}\right]\right)=\mathbf{J}_{\mathrm{AC}, n_{1}} \operatorname{cov}\left(\operatorname{vec}\left(\hat{\mathbf{\Lambda}}_{1 \mid 2 i-1}\right)\right) \mathbf{J}_{\mathrm{AC}, n_{2}}^{\mathrm{T}},
$$

where the subscripts $n_{1}$ and $n_{2}$ indicate that the system matrices can have different model orders. This is an attractive feature of the method since when e.g. the stabilization diagram technique is employed, the different modes are not necessarily estimated at the same model order. Explicit expressions for the Jacobians $\mathbf{J}_{\mathrm{AC}, n_{1}}$ and $\mathbf{J}_{\mathrm{AC}, n_{2}}$ are provided in [1, Sec. 5], and an optimized computer implementation is presented in [19].

The covariance matrices of the modal characteristics can be found in a similar way as the covariance matrices of the system matrices. A first-order sensitivity analysis relating changes in the system matrices to changes in the estimates of the eigenfrequency $f_{k}$, damping ratio $\xi_{k}$ and mode shape $\phi_{k}$ of mode $k$ leads to the corresponding Jacobian:

$$
\Delta\left[\begin{array}{c}
f_{k} \\
\xi_{k} \\
\mathcal{R}\left(\phi_{k}\right) \\
\mathcal{I}\left(\phi_{k}\right)
\end{array}\right]=\mathbf{J}_{k}\left[\begin{array}{c}
\operatorname{vec}(\Delta \mathbf{A}) \\
\operatorname{vec}(\Delta \mathbf{C})
\end{array}\right]
$$

where $\mathcal{R}($ ) denotes, as before, the real part of a variable, vector or matrix, and $\mathcal{I}()$ denotes the imaginary part. The expression for $\mathbf{J}_{k}$ is detailed in the Appendix, where it is also indicated how $\mathbf{J}_{k}$ changes when the mode shape is rescaled. The full covariance matrix between the modal characteristics of any two modes $k$ and $l$ can be obtained, up to first order, from

$$
\operatorname{cov}\left(\left[\begin{array}{c}
\hat{f}_{k} \\
\hat{\xi}_{k} \\
\mathcal{R}\left(\hat{\phi}_{k}\right) \\
\mathcal{I}\left(\hat{\phi}_{k}\right)
\end{array}\right],\left[\begin{array}{c}
\hat{f}_{l} \\
\hat{\xi}_{l} \\
\mathcal{R}\left(\hat{\phi}_{l}\right) \\
\mathcal{I}\left(\hat{\phi}_{l}\right)
\end{array}\right]\right)=\mathbf{J}_{k} \mathbf{J}_{\mathrm{AC}, n_{1}} \operatorname{cov}\left(\operatorname{vec}\left(\hat{\boldsymbol{\Lambda}}_{1 \mid 2 i-1}\right)\right) \mathbf{J}_{\mathrm{AC}, n_{2}}^{\mathrm{T}} \mathbf{J}_{l}^{\mathrm{T}}
$$

Note again that the system descriptions from which modes $k$ and $l$ are estimated do not need to have the same model order $n$. Although this covariance matrix has been obtained from a first-order sensitivity approach, numerical verification studies indicate a high level of accuracy, also for the modal damping ratios of lightly damped modes, for which the relative uncertainty of the estimates is large by nature $[1,5]$.

For the application of Eq. (22), the covariance matrix of vec $\left(\hat{\boldsymbol{\Lambda}}_{1 \mid 2 i-1}\right)$ is needed. It can be obtained as follows [1]. By dividing the available data into $n_{\mathrm{b}}$ blocks containing $N_{\mathrm{b}}$ samples each, one has:

$$
\hat{\boldsymbol{\Lambda}}_{l}=\frac{1}{n_{\mathrm{b}}} \sum_{b=1}^{n_{\mathrm{b}}} \hat{\boldsymbol{\Lambda}}_{l, b}
$$

where

$$
\hat{\boldsymbol{\Lambda}}_{l, b}:=\frac{1}{N_{\mathrm{b}}} \sum_{k=N_{\mathrm{b}}(b-1)+1}^{N_{\mathrm{b}} b} \mathbf{y}_{k+l} \mathbf{y}_{k}^{\mathrm{ref}^{\mathrm{T}}}
$$


Note that the expressions (16) and (23) for $\hat{\boldsymbol{\Lambda}}_{l}$ are completely equivalent. If the block length $N_{\mathrm{b}}$ is large, the different block estimates $\hat{\Lambda}_{l, b}$ are approximately statistically independent, so that $\hat{\boldsymbol{\Lambda}}_{l}$ is the sample mean of $n_{\mathrm{b}}$ independent random matrices $\hat{\boldsymbol{\Lambda}}_{l, b}$. Consequently, the covariance matrix between all elements of $\hat{\boldsymbol{\Lambda}}_{1 \mid 2 i-1}$ can be estimated as the variance of the sample mean:

$$
\operatorname{cov}\left(\operatorname{vec}\left(\hat{\boldsymbol{\Lambda}}_{1 \mid 2 i-1}\right)\right) \approx \frac{1}{n_{\mathrm{b}}^{2}} \sum_{b=1}^{n_{\mathrm{b}}} \operatorname{vec}\left(\hat{\boldsymbol{\Lambda}}_{1 \mid 2 i-1, b}-\hat{\boldsymbol{\Lambda}}_{1 \mid 2 i-1}\right)\left(\operatorname{vec}\left(\hat{\boldsymbol{\Lambda}}_{1 \mid 2 i-1, b}-\hat{\boldsymbol{\Lambda}}_{1 \mid 2 i-1}\right)\right)^{\mathrm{T}}
$$

Note that, when the total number of data samples is fixed, the choice of the number of blocks $n_{\mathrm{b}}$ requires a trade-off: the different blocks should be sufficiently large for guaranteeing statistical independency between them, which requires a relatively small value of $n_{\mathrm{b}}$, but the sample (co)variance only approximates the true variance well when the number of blocks $n_{\mathrm{b}}$ is relatively large. The values that are employed in this paper reflect this trade-off. It has been verified that the reported results are not very sensitive to the particular value of $n_{\mathrm{b}}$ that is adopted.

\subsection{System matrices and modal characteristics: probability distribution of the estimates}

From the central limit theorem, it follows immediately that the elements of vec $\left(\hat{\Lambda}_{1 \mid 2 i-1}\right)$ are asymptotically jointly normally distributed, because $\hat{\boldsymbol{\Lambda}}_{1 \mid 2 i-1}$ is the mean of a sequence of independent samples. Since linear combinations of normally distributed random variables are normally distributed, it follows that the estimates of the modal characteristics are also asymptotically normally distributed up to first order accuracy [5]. It should be kept in mind though that these results are asymptotic in the number of data samples, and deviations from the normal distribution have been observed for short data sequences [27].

\section{First experimental validation: damaged prestressed concrete bridge}

In this section, the uncertainty quantification approach for operational modal analysis based on SSI-cov as outlined above is validated by application to vibration data collected on a three-span prestressed concrete roadway bridge for which the dynamic response has been collected in 46 degrees of freedom in 36 different measurement setups. Processing each setup separately results in 36 different estimates for the eigenfrequencies and damping ratios of interest, and the sample variances and sample cumulative distribution functions computed from the set of 36 estimates are compared with the estimates resulting from the algorithm for each setup. The variability in the estimation accuracy of all modal characteristics within a single setup and between different setups is investigated as well.

\subsection{Description of the structure and the ambient vibration test}

A series of inspections of the former bridge of Tilff, Belgium, performed in the period 2003-2005, revealed that the structural integrity of this three-cell box girder bridge was endangered by corrosion. Due to an inefficient drainage system, water accumulated in the hollow cells and severe corrosion of the prestressing strands took place. The resulting reduction of the prestressing force jeopardized the further utilization of the bridge. In 2005, a Bailey bridge was mounted on top of the bridge deck in order to ensure a safe passage for road traffic on the bridge. As the original bridge deck continued to degrade, it was completely demolished in 2007.

On 24 and 25 June 2004, an ambient vibration survey was carried out on the original bridge by the Ministry of Equipment and Transport of the Walloon Region, Belgium, using a low-cost data acquisition system with four channels (Fig. 1). With this system, vertical accelerations and longitudinal strains were measured in 36 different setups. In each setup, two vertical accelerations on top of the bridge deck were measured using accelerometers. One accelerometer was used as a reference (at location 18, see Fig. 2), for gluing the partial mode shapes obtained in the different setups together, while the other accelerometer was used for scanning the bridge deck. The acceleration numbers in Fig. 2 denote the position of the roving accelerometer in the corresponding setup. In each measurement setup, the dynamic axial strains at the top and bottom of a particular section were measured with two optical fibres. The five sections for which the strains were measured are indicated in Fig. 2 with Roman numbers. The strains at section I were measured in setups 1 to 8 , the strains at section II in setups 9 to 16 , the strains at section III in setups 17 to 24, the strains at section IV in setups 25 to 29 , and the strains in section V in setups 30 to 36. Strains and accelerations have been measured simultaneously, using the same data acquisition system. 


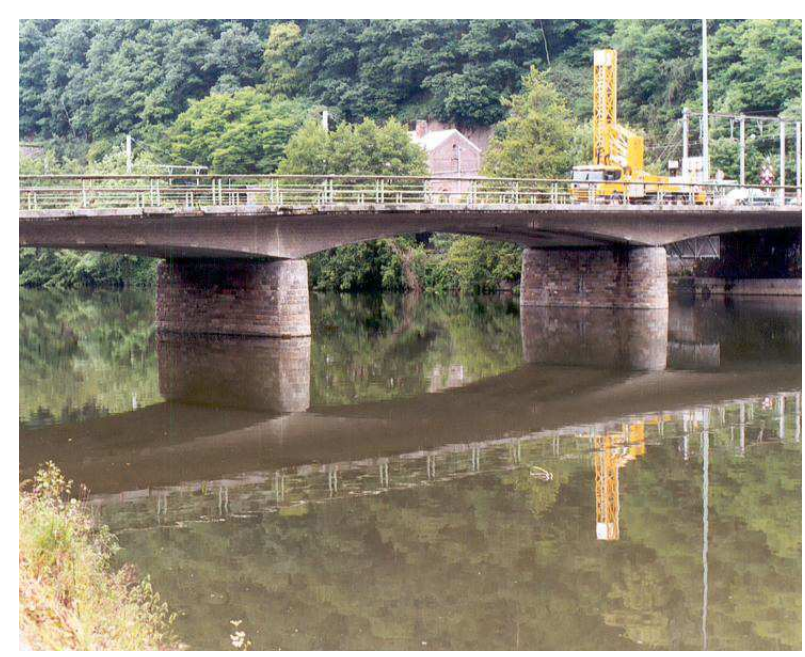

(a)

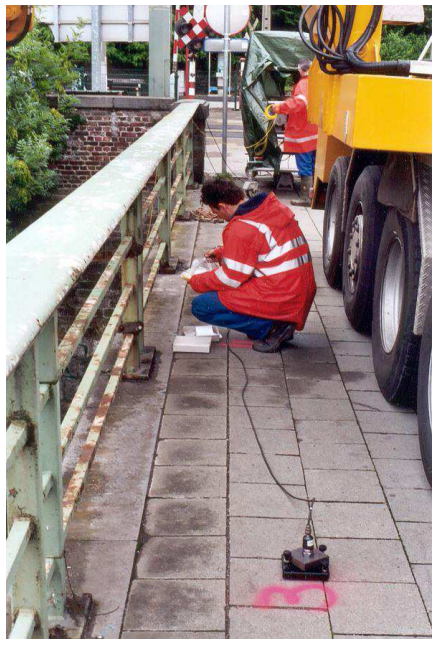

(b)

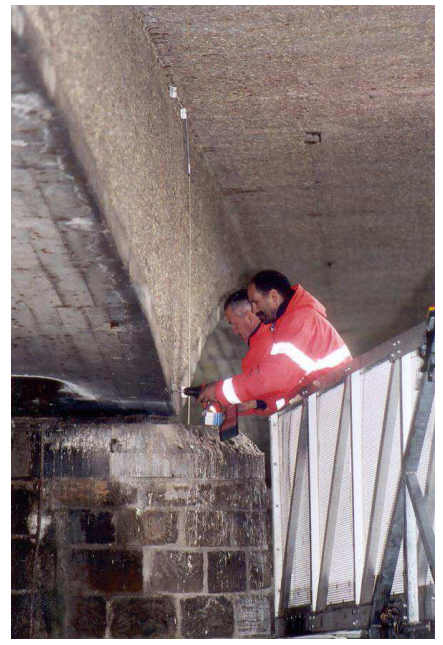

(c)

Figure 1: The Tilff bridge during ambient vibration testing: (a) overview, (b) accelerometers, (c) fibre optic sensors.

Downstream

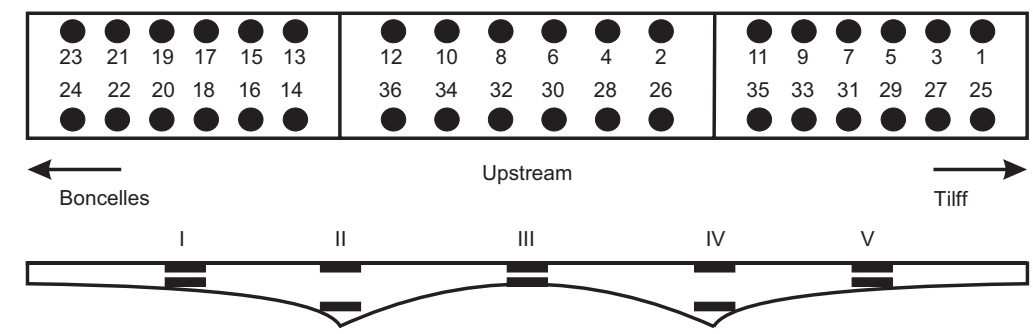

Figure 2: Tilff bridge: top view with the location of the measured vertical accelerations (circles) and side view with the location of the measured longitudinal strains (rectangles).

For each setup, a total of 48000 data samples were gathered at a sampling frequency of $200 \mathrm{~Hz}$, so the measurement duration for each setup was 4 minutes. After removing the static (or DC) offset from the measured signals, the data were low-pass filtered by an eight-order Chebyshev Type I filter with cut-off frequency at $26.7 \mathrm{~Hz}$ in both the forward and reverse directions to remove all phase distortion, before re-sampling them at a sampling rate of $66 \mathrm{~Hz}$.

A unique feature of this experiment was that dynamic strain measurements were included in the ambient vibration test, next to the more common acceleration measurements, so modal strains and modal curvatures could be identified directly. The knowledge of these modal strains was important for the deterministic damage identification study that was performed shortly after the experiment [28], because modal strains and the related modal curvatures tend to be much more sensitive to local damage than other modal characteristics [29]. Modal curvatures can also be derived from measured mode shapes using numerical differentiation, however, this is a numerically ill-conditioned procedure that often leads to inaccurate estimates, see, e.g., [28, Table 2].

\subsection{Modal characteristics: point estimates}

The SSI-cov algorithm was used for computing the modal characteristics and their standard deviations. The number of block rows in the correlation matrix $\mathbf{L}_{1 \mid i}$ was set to $i=40$, and the number of data blocks employed for estimating the variance of the output correlation estimates was set to $n_{\mathrm{b}}=62$, so each block consists of $N_{\mathrm{b}}=256$ samples which amounts to $3.9 \mathrm{~s}$ of data per block. For the construction of the stabilization diagrams, a model order range from $n=2$ to $n=40$ was chosen, with increments of 2 . The acceleration output that was common to all setups was employed as a reference output in the algorithm. 
Mode $1-4.323 \mathrm{~Hz}-1.3 \%$

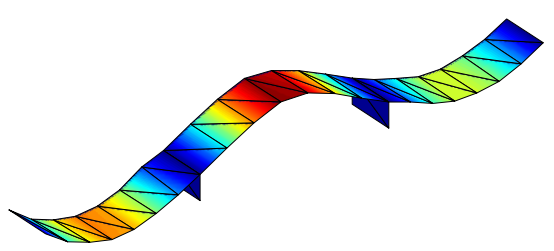

Mode $3-8.683 \mathrm{~Hz}-2.6 \%$

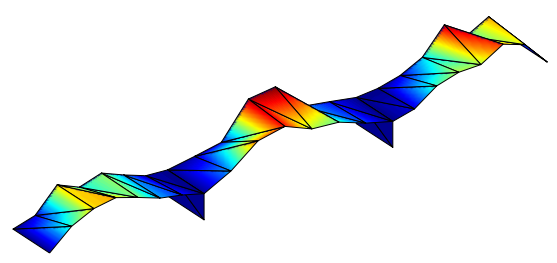

Mode $2-6.502 \mathrm{~Hz}-1.6 \%$

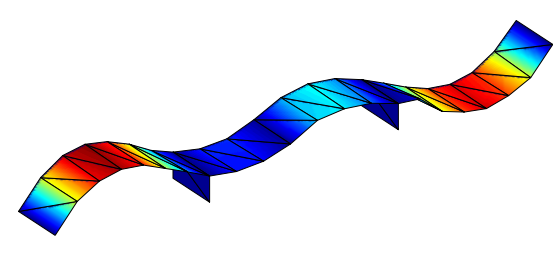

Mode $4-10.403 \mathrm{~Hz}-1.7 \%$

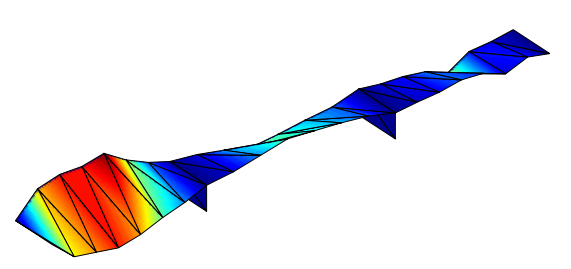

Figure 3: Tilff bridge: identified eigenfrequencies, damping ratios and displacement mode shapes.

Four different modes were found in all 36 setups. The point estimates of the eigenfrequencies, damping ratios, and displacement mode shapes, are shown in Fig. 3. The identified mode shapes of modes 1 and 2 are the smoothest, while the other two mode shapes, in particular the one of mode 3, are less smooth. This suggests that the estimates of mode shapes 1 and 2 are more accurate than the other ones. Therefore, the estimates of mode shapes 3 and 4 have not been used previously in a deterministic damage identification study [28]. These facts, together with the limited number of channels, reference sensors and data samples that were available, render the question for uncertainty quantification very relevant. In the next sections, the uncertainty of the different modal estimates will be assessed and compared.

\subsection{Uncertainty quantification of the estimated modal characteristics}

The eigenfrequency and damping ratio estimates and their $95 \%$ confidence intervals, obtained for each of the 36 different setups, are shown in Fig. 4. The estimates of modes 1 and 2 are much more accurate than the estimates of modes 3 and 4 . This complies with the observation made in the previous section that the mode shapes of modes 1 and 2 are smoother than the mode shapes of modes 3 and 4 . The accuracy of the estimated values changes considerably from setup to setup. This is particularly true for mode 4 , which does not seem to be well excited by the ambient forces in some particular setups. It may be noted that the $95 \%$ confidence intervals for the damping ratio estimates sometimes encompass negative damping ratios, although for most structures positive damping ratios are expected on physical grounds. This is however in agreement with the fact that the SSI-cov algorithm may yield negative modal damping estimates, as the identified modes are not constrained to be stable.

Table 1 compares the sample standard deviations of the eigenfrequency and damping ratio estimates, obtained over the 36 different setups, with the single-batch standard deviation estimates, computed in each setup. When the excitation would be stationary across all setups and the structure itself would not change, both standard deviations should be similar, despite the fact that the measurement layout changes between different setups. This is because in all setups, all outputs are correlated to the same reference outputs in the subspace algorithm (see Eq. (9)), and therefore the basic information that is employed during the identification - in other words, the data subspace - would be the same for all setups under the stated assumptions.

The eigenfrequency and damping ratio values that are listed in the first row of Table 1 are computed as the sample mean of the eigenfrequency estimates $\hat{f}_{k, s}$ and damping ratio estimates $\hat{\xi}_{k, s}$ which are available for every setup $s$ :

$$
\mu_{\mathrm{samp}}\left(\hat{f}_{k}\right):=\frac{1}{n_{\mathrm{s}}} \sum_{s=1}^{n_{\mathrm{s}}} \hat{f}_{k, s} \quad \text { and } \quad \mu_{\mathrm{samp}}\left(\hat{\xi}_{k}\right):=\frac{1}{n_{\mathrm{s}}} \sum_{s=1}^{n_{\mathrm{s}}} \hat{\xi}_{k, s}
$$



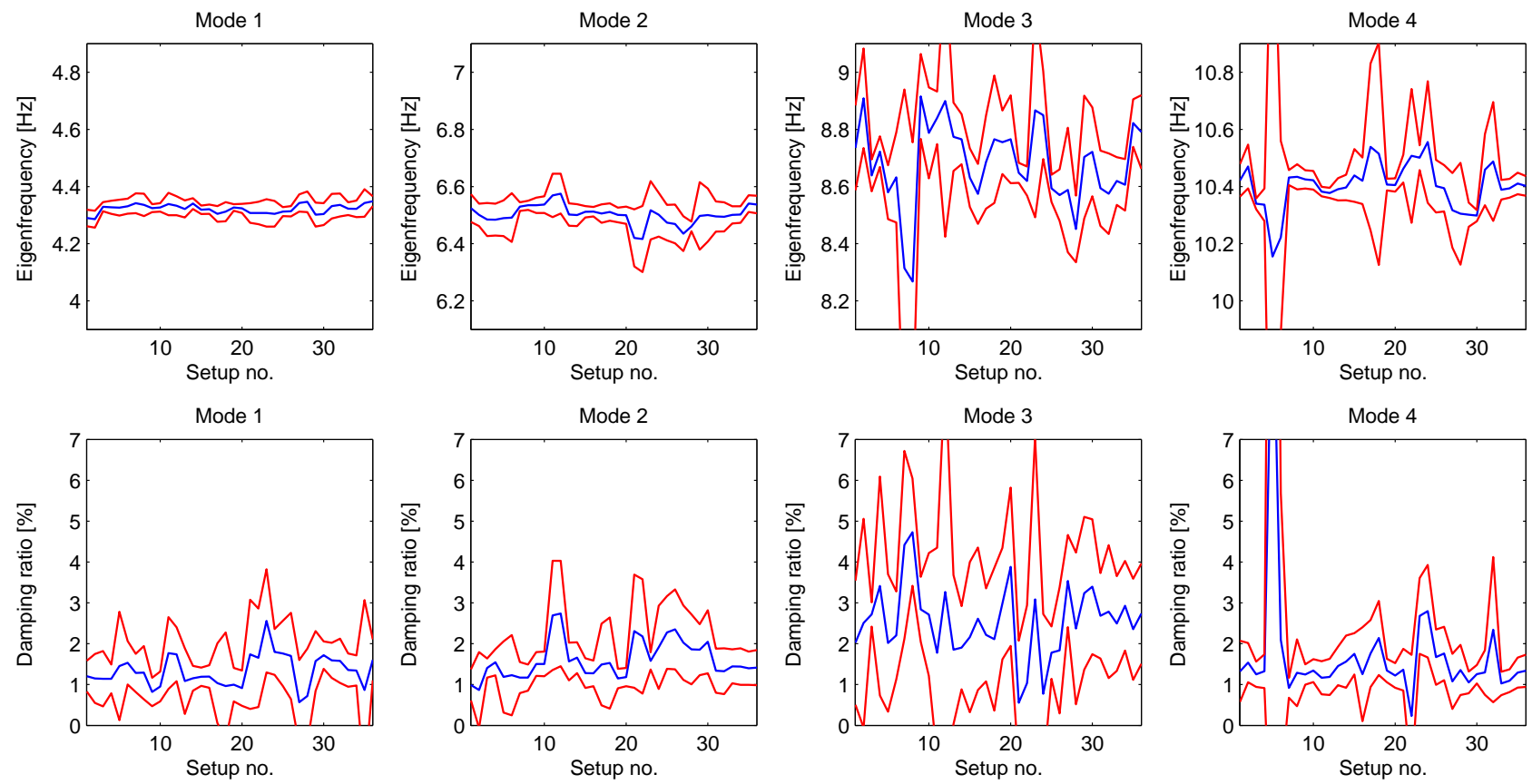

Figure 4: Tilff bridge: estimated eigenfrequencies, estimated damping ratios, and the corresponding $95 \%$ confidence intervals for all setups.

where $n_{\mathrm{s}}$ denotes the number of setups; $n_{\mathrm{s}}=36$ in the present case. The sample standard deviations of the eigenfrequency estimates $\hat{f}_{k, s}$ and damping ratio estimates $\hat{\xi}_{k, s}$ are computed from

$$
\sigma_{\mathrm{samp}}\left(\hat{f}_{k}\right):=\frac{1}{n_{\mathrm{s}}} \sqrt{\sum_{s=1}^{n_{\mathrm{s}}}\left(\hat{f}_{k, s}-\mu_{\mathrm{samp}}\left(\hat{f}_{k}\right)\right)^{2}} \quad \text { and } \quad \sigma_{\mathrm{samp}}\left(\hat{\xi}_{k}\right):=\frac{1}{n_{\mathrm{s}}} \sqrt{\sum_{s=1}^{n_{\mathrm{s}}}\left(\hat{\xi}_{k, s}-\mu_{\mathrm{samp}}\left(\hat{\xi}_{k}\right)\right)^{2}}
$$

respectively. These sample standard deviations are shown in the second row of Table 1. The sample mean of the estimated standard deviations of the eigenfrequency estimates, $\hat{\sigma}\left(\hat{f}_{k, s}\right)$, and the estimated standard deviations of the damping ratio estimates, $\hat{\sigma}\left(\hat{\xi}_{k, s}\right)$, which are also available for every setup $s$, are computed from

$$
\mu_{\mathrm{samp}}\left(\hat{\sigma}\left(\hat{f}_{k}\right)\right):=\frac{1}{n_{\mathrm{s}}} \sum_{s=1}^{n_{\mathrm{s}}} \hat{\sigma}\left(\hat{f}_{k, s}\right) \quad \text { and } \quad \mu_{\mathrm{samp}}\left(\hat{\sigma}\left(\hat{\xi}_{k}\right)\right):=\frac{1}{n_{\mathrm{s}}} \sum_{s=1}^{n_{\mathrm{s}}} \hat{\sigma}\left(\hat{\xi}_{k, s}\right)
$$

They are shown in the third row of Table 1. The sample standard deviations (second row of Table 1) agree very well with the mean of the estimated standard deviations (third row of Table 1) when considering the variability (or more precisely, the sample standard deviation) of the estimated standard deviations over the different setups (fourth row of Table 1).

Fig. 5 compares the empirical probability distributions of the eigenfrequency and damping ratio estimates over the 36 different setups with a normal distribution, constructed from the sample means $\mu_{\text {samp }}\left(\hat{f}_{k}\right)$ and $\mu_{\text {samp }}\left(\hat{\xi}_{k}\right)$ and the sample variances $\sigma_{\text {samp }}\left(\hat{f}_{k}\right)$ and $\sigma_{\text {samp }}\left(\hat{\xi}_{k}\right)$. A reasonable agreement is observed in all cases.

Fig. 6 plots the modal displacements, obtained after merging the different setups together by scaling the modal displacement in the reference degree of freedom to one for all setups. The $99 \%$ confidence intervals are also plotted. Not only does the uncertainty of the modal displacements vary considerably from one mode to another - mode shapes 1 and 2 are again much more accurate than mode shapes 3 and 4 -, the uncertainty of the displacement estimates within a single mode shape also varies considerably. This is clearly visible for modes 1 and 2 , where the less smooth parts of the mode shapes have a much larger uncertainty than the smooth parts, as one would expect. Fig. 7 plots the identified modal strains that result from the strain measurements at the top and bottom of five different sections as detailed previously (the section 
Table 1: Eigenfrequency estimates $\hat{f}_{k}$ and damping ratio estimates $\hat{\xi}_{k}$ of the Tilff bridge: sample mean $\mu_{\text {samp }}$ and sample standard deviation $\sigma_{\text {samp }}$ of the point estimates, computed over the 36 different setups, together with the mean of the estimated standard deviations for each setup $\mu_{\mathrm{samp}}(\hat{\sigma})$ and the related standard deviation $\sigma_{\text {samp }}(\hat{\sigma})$.

\begin{tabular}{llrrrr}
\hline Mode no. & & 1 & 2 & 3 & 4 \\
\hline$\mu_{\text {samp }}\left(\hat{f}_{k}\right)$ & {$[\mathrm{Hz}]$} & 4.323 & 6.502 & 8.683 & 10.403 \\
$\sigma_{\text {samp }}\left(\hat{f}_{k}\right)$ & {$[\mathrm{Hz}]$} & 0.016 & 0.034 & 0.149 & 0.083 \\
$\mu_{\text {samp }}\left(\hat{\sigma}\left(\hat{f}_{k}\right)\right)[\mathrm{Hz}]$ & 0.015 & 0.026 & 0.081 & 0.065 \\
$\sigma_{\text {samp }}\left(\hat{\sigma}\left(\hat{f}_{k}\right)\right)[\mathrm{Hz}]$ & 0.005 & 0.015 & 0.065 & 0.118 \\
\hline
\end{tabular}

\begin{tabular}{llcccc}
\hline Mode no. & & 1 & 2 & 3 & 4 \\
\hline$\mu_{\text {samp }}\left(\hat{\xi}_{k}\right)$ & {$[\%]$} & 1.34 & 1.61 & 2.56 & 1.72 \\
$\sigma_{\text {samp }}\left(\hat{\xi}_{k}\right)$ & {$[\%]$} & 0.40 & 0.46 & 0.89 & 1.70 \\
$\mu_{\text {samp }}\left(\hat{\sigma}\left(\hat{\xi}_{k}\right)\right)[\%]$ & 0.36 & 0.33 & 0.86 & 0.54 \\
$\sigma_{\text {samp }}\left(\hat{\sigma}\left(\hat{\xi}_{k}\right)\right)[\%]$ & 0.21 & 0.19 & 0.45 & 1.24 \\
\hline
\end{tabular}

numbers correspond to those of Fig. 2). Also here the accuracy of the estimates varies considerable from setup to setup, and the corresponding variance estimates make it possible to take these differences into account. Axial modal strains are only relevant for the bending modes, i.e., for modes 1 to 3 , not for the fourth mode, which is a torsion mode.

\subsection{Unweighted versus weighted averaging}

To conclude this first validation study, the accuracy of the averaged eigenfrequency and damping ratio estimates, which are listed in Table 1 as $\mu_{\text {samp }}\left(\hat{f}_{k}\right)$ and $\mu_{\text {samp }}\left(\hat{\xi}_{k}\right)$, respectively, is investigated. The eigenfrequency and damping ratio estimates are statistically independent for different setups $s$, because the data from which the estimates are computed are also different. The standard deviations of these unweighted averages can then be estimated from

$$
\hat{\sigma}\left(\mu_{\mathrm{samp}}\left(\hat{f}_{k}\right)\right)=\frac{1}{n_{\mathrm{s}}} \sqrt{\sum_{s=1}^{n_{\mathrm{s}}} \hat{\sigma}^{2}\left(\hat{f}_{k, s}\right)} \quad \text { and } \quad \hat{\sigma}\left(\mu_{\mathrm{samp}}\left(\hat{\xi}_{k}\right)\right)=\frac{1}{n_{\mathrm{s}}} \sqrt{\sum_{s=1}^{n_{\mathrm{s}}} \hat{\sigma}^{2}\left(\hat{\xi}_{k, s}\right)} .
$$
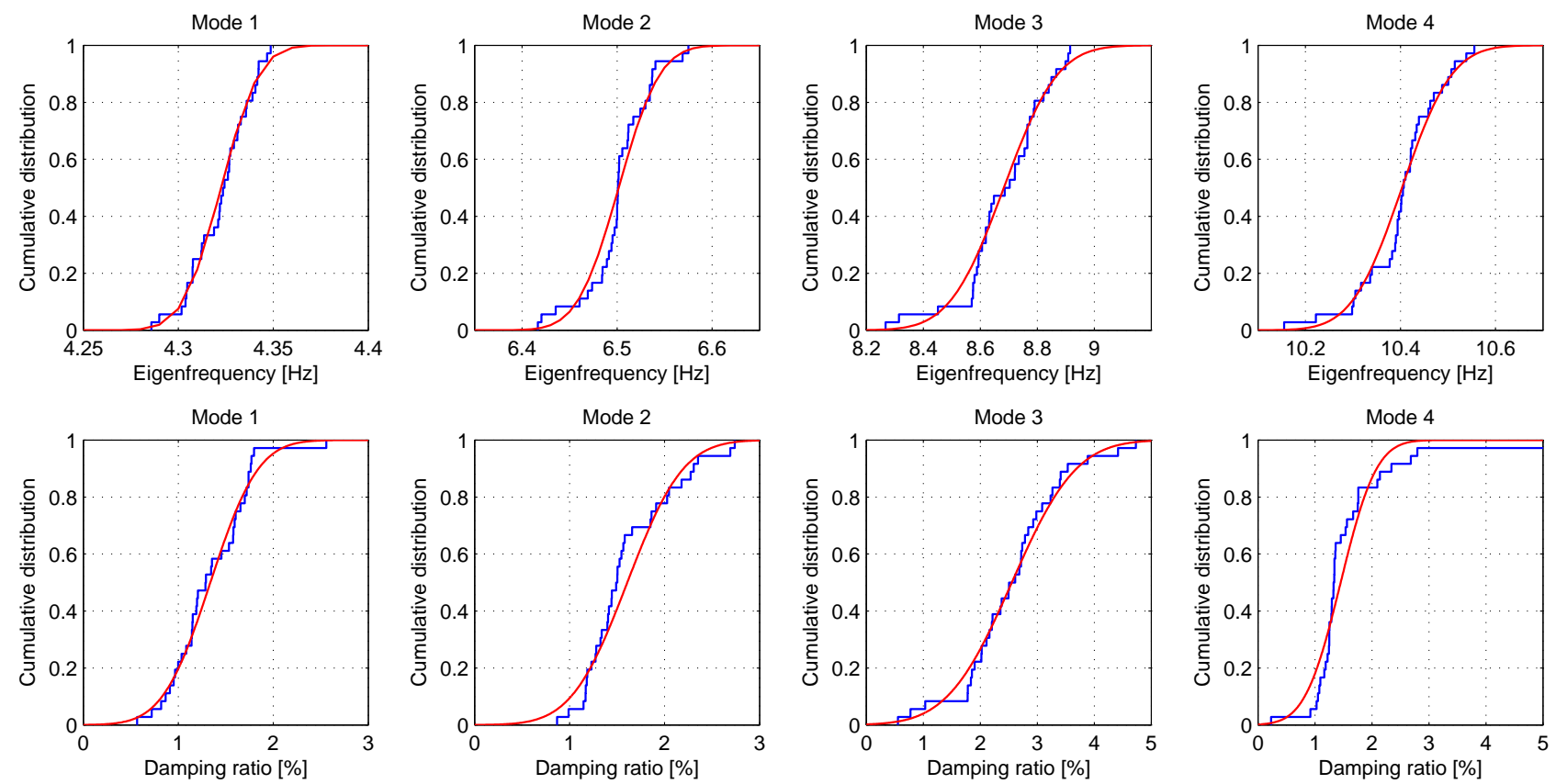

Figure 5: Tilff bridge: Empirical cumulative distribution function of the eigenfrequencies and damping ratios over the 36 different setups, and normal cumulative distribution constructed from the sample mean and sample variance. 

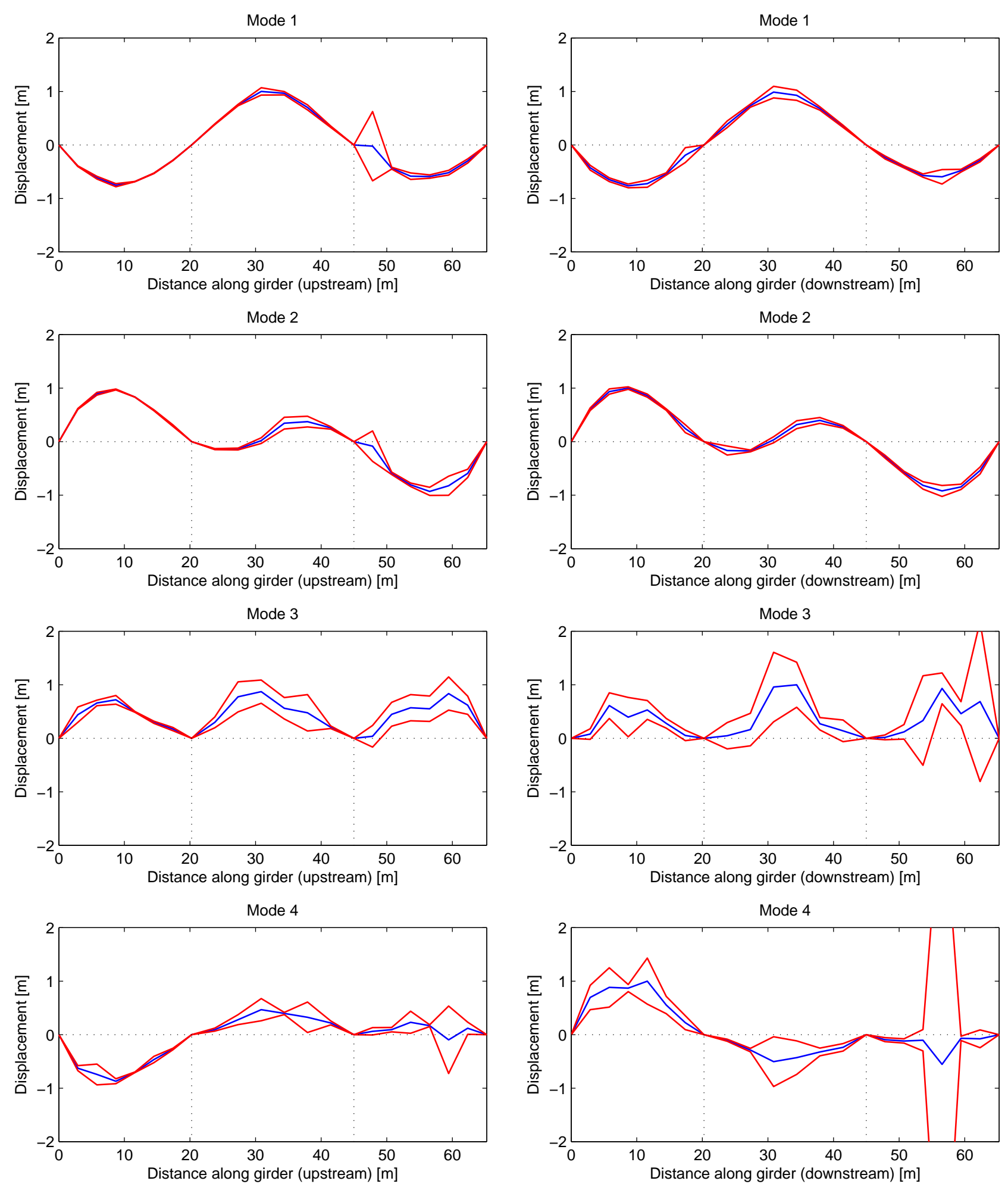

Figure 6: Tilff bridge: point estimates of the modal displacements and $99 \%$ confidence intervals. 

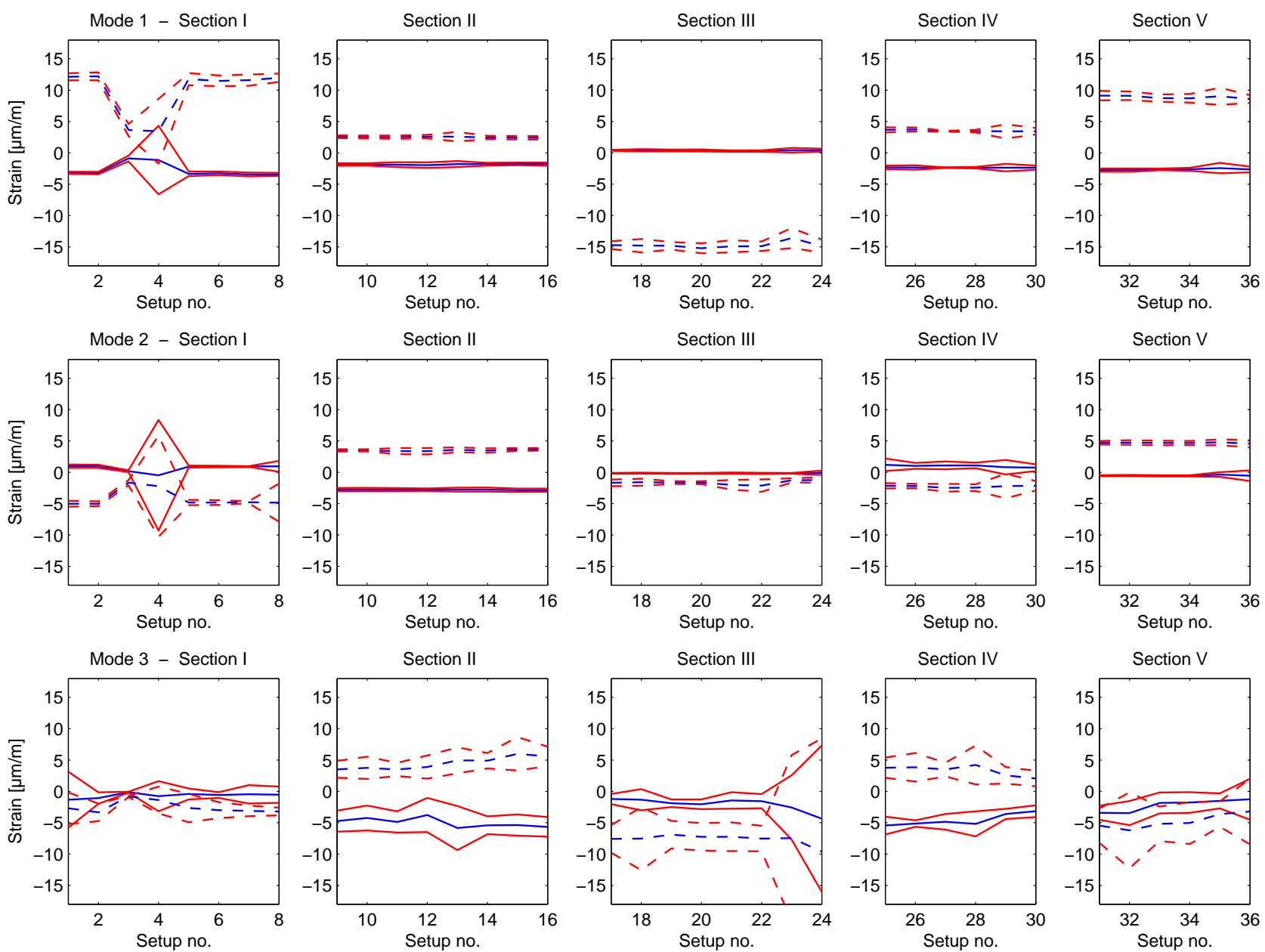

Figure 7: Tilff bridge: point estimates of the modal strains at the upper side (solid lines) and lower side (dashed lines) of the five cross sections, and corresponding $99 \%$ confidence intervals. 
Note that $\hat{\sigma}\left(\mu_{\text {samp }}\left(\hat{f}_{k}\right)\right)$ is different from $\sigma_{\text {samp }}\left(\hat{f}_{k}\right)$, which was defined in $(27): \hat{\sigma}\left(\mu_{\text {samp }}\left(\hat{f}_{k}\right)\right)$ denotes the estimated variance of the sample mean, while $\sigma_{\text {samp }}\left(\hat{f}_{k}\right)$ denotes the estimated variance of the entire statistical ensemble.

An alternative for the unweighted sample mean is the maximum likelihood (ML) estimate for the mean. This is a weighted estimate, where the weights are inversely proportional to the variances for each setup:

$$
\mu_{\mathrm{ML}}\left(\hat{f}_{k}\right):=\frac{\sum_{s=1}^{n_{\mathrm{s}}} \frac{\hat{f}_{k, s}}{\hat{\sigma}^{2}\left(\hat{f}_{k, s}\right)}}{\sum_{s=1}^{n_{\mathrm{s}}} \frac{1}{\hat{\sigma}^{2}\left(\hat{f}_{k, s}\right)}} \quad \text { and } \quad \mu_{\mathrm{ML}}\left(\hat{\xi}_{k}\right):=\frac{\sum_{s=1}^{n_{\mathrm{s}}} \frac{\hat{\xi}_{k, s}}{\hat{\sigma}^{2}\left(\hat{\xi}_{k, s}\right)}}{\sum_{s=1}^{n_{\mathrm{s}}} \frac{1}{\hat{\sigma}^{2}\left(\hat{\xi}_{k, s}\right)}}
$$

The related standard deviations can be estimated as

$$
\hat{\sigma}\left(\mu_{\mathrm{ML}}\left(\hat{f}_{k}\right)\right)=\sqrt{\frac{1}{\sum_{s=1}^{n_{\mathrm{s}}} \frac{1}{\hat{\sigma}^{2}\left(\hat{f}_{k, s}\right)}}} \quad \text { and } \quad \hat{\sigma}\left(\mu_{\mathrm{ML}}\left(\hat{\xi}_{k}\right)\right)=\sqrt{\frac{1}{\sum_{s=1}^{n_{\mathrm{s}}} \frac{1}{\hat{\sigma}^{2}\left(\hat{\xi}_{k, s}\right)}}},
$$

respectively. The unweighted and the maximum likelihood averages of the eigenfrequency and damping ratio estimates of the Tilff bridge are listed in Table 2. The standard deviations that correspond to these averages are also listed. By comparing the standard deviations of the unweighted and weighted averages, it can be observed that taking the uncertainty of the point estimates for each measurement setup into account leads to more accurate results when averaging over all setups. The difference is especially striking for the fourth mode, for which the accuracy of the frequency and damping ratio estimates varies heavily between the different setups, as was seen previously in Fig. 4. For this particular mode, the unweighted mean of the eigenfrequency estimates has a standard deviation of $\hat{\sigma}\left(\mu_{\text {samp }}\left(\hat{f}_{4}\right)\right)=0.0222$ while the weighted mean only has a standard deviation of $\hat{\sigma}\left(\mu_{\mathrm{ML}}\left(\hat{f}_{k}\right)\right)=0.0031$. Similarly, the unweighted mean of the damping ratio estimates for the fourth mode has a standard deviation of $\hat{\sigma}\left(\mu_{\text {samp }}\left(\hat{\xi}_{k}\right)\right)=0.223$, while the weighted mean has a standard deviation of $\hat{\sigma}\left(\mu_{\mathrm{ML}}\left(\hat{\xi}_{k}\right)\right)=0.035$, which is much lower.

Table 2: Tilff bridge: unweighted and maximum likelihood averages of the eigenfrequency estimates (in $\mathrm{Hz}$ ) and damping ratio estimates (in \% of critical), and corresponding standard deviations.

\begin{tabular}{lrrrr}
\hline Mode no. & 1 & 2 & 3 & 4 \\
\hline$\mu_{\text {samp }}\left(\hat{f}_{k}\right)$ & 4.3224 & 6.5016 & 8.6827 & 10.4035 \\
$\hat{\sigma}\left(\mu_{\text {samp }}\left(\hat{f}_{k}\right)\right)$ & 0.0026 & 0.0049 & 0.0172 & 0.0222 \\
\hline$\mu_{\mathrm{ML}}\left(\hat{f}_{k}\right)$ & 4.3238 & 6.5105 & 8.6639 & 10.3890 \\
$\hat{\sigma}\left(\mu_{\mathrm{ML}}\left(\hat{f}_{k}\right)\right)$ & 0.0019 & 0.0026 & 0.0076 & 0.0031 \\
\hline
\end{tabular}

\begin{tabular}{lcccc}
\hline Mode no. & 1 & 2 & 3 & 4 \\
\hline$\mu_{\text {samp }}\left(\hat{\xi}_{k}\right)$ & 1.338 & 1.607 & 2.557 & 1.720 \\
$\hat{\sigma}\left(\mu_{\mathrm{samp}}\left(\hat{\xi}_{k}\right)\right)$ & 0.069 & 0.063 & 0.162 & 0.223 \\
\hline$\mu_{\mathrm{ML}}\left(\hat{\xi}_{k}\right)$ & 1.266 & 1.402 & 2.521 & 1.255 \\
$\hat{\sigma}\left(\mu_{\mathrm{ML}}\left(\hat{\xi}_{k}\right)\right)$ & 0.040 & 0.035 & 0.108 & 0.035 \\
\hline
\end{tabular}

It should be kept in mind though that the variances of the unweighted and weighted averages that are obtained in this way, only take intra-setup variability into account, but not inter-setup variability. For example, it is known that eigenfrequencies of civil structures may depend on environmental factors such as temperature [30]. If the temperature is fairly constant within each setup, but varies between different setups, then the point estimates of the eigenfrequencies that are obtained with SSI for each setup, may vary considerably between setups, while the estimated standard deviations that correspond to each point estimate may be very low. Similarly, damping ratios of civil structures may depend on the amplitude of excitation [31]. If this is the case, and the excitation amplitude varies largely between different setups but much less within setups, then this variability will not be accounted for in the averaged values.

\section{Second experimental validation: mid-rise office building}

This section presents a second experimental validation of the method for estimating the variance of modal characteristics that are identified with SSI. The method is applied to the operational modal analysis of a mid-rise building, for which ambient vibration data have been collected in 252 degrees of freedom in 10 different setups. 


\subsection{Description of the structure and the ambient vibration test}

The IMEC Tower is a recent, $73 \mathrm{~m}$ high tower building in Leuven, Belgium, designed by Baumschlager-Eberle. It consists of 17 storeys, including four underground storeys and a technical storey on the roof. The construction started in May 2011, with Vanhout/Besix as main contractor and Jan Van Aelst as structural engineering office. The tower offers office space for 450 employees of IMEC, one of the world's largest research centers for micro- and nano-electronics. On 13 June 2013, when the main structure of the tower was completed, an output-only modal test was performed with the aim of identifying eigenfrequencies, damping ratios and mode shapes of the tower's first few global modes, that are excited mainly by ambient wind loading (Fig. 8). Acceleration data have been collected at all floors by twelve wireless GeoSIG GMS-18 units, each containing a high-sensitivity triaxial MEMS acceleration sensor. The test was performed in ten measurement setups. In each setup, three floors were instrumented with four units each. The positions of the units at the top office floor were kept fixed, while the other units were roving from floor to floor. The acceleration data were originally sampled at $200 \mathrm{~Hz}$ during 20 minutes for each measurement setup.
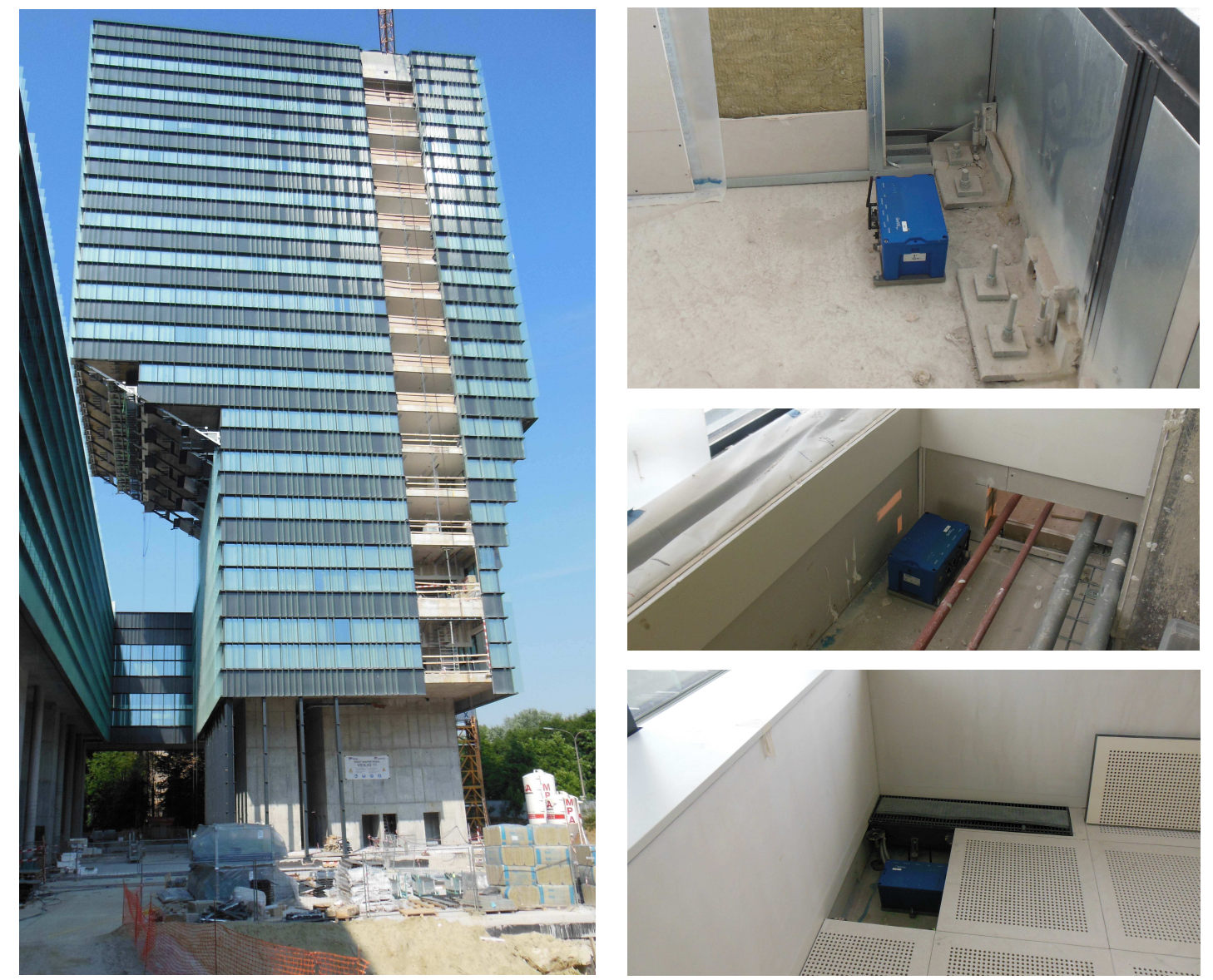

Figure 8: IMEC Tower: overview during construction in June 2013, and wireless triaxial accelerometers, positioned onto the concrete floor.

The measured signals were digitally low-pass filtered by an eighth-order Chebyshev type I filter with a cutoff frequency of $8 \mathrm{~Hz}$ in both the forward and reverse direction to remove all phase distortion, and then re-sampled at $20 \mathrm{~Hz}$. Subsequently, the signals were high-pass filtered by a fourth-order Butterworth filter with a cutoff frequency of $0.15 \mathrm{~Hz}$, again in both the forward and reverse direction, in order to remove the quasi-static drift. 


\subsection{Modal characteristics: point estimates}

The SSI-cov algorithm was then applied to the IMEC Tower data. The number of block rows in the correlation matrix $\mathbf{L}_{1 \mid i}$ was taken as $i=50$, and the number of data blocks employed for the variance estimation as $n_{\mathrm{b}}=50$, so each block consists of $N_{\mathrm{b}}=478$ samples which amounts to $23.9 \mathrm{~s}$ of data per block. For the construction of the stabilization diagrams, a model order range from $n=2$ to $n=80$ in increments of 2 was considered. The acceleration outputs common to all setups were employed as reference outputs in the algorithm.

Six different modes were found in all ten setups. The point estimates of their eigenfrequencies, damping ratios and mode shapes are shown in Fig. 9. The modal displacements at and below ground level are small, which indicates that for dynamic modeling, the building can be considered as clamped at the ground floor. When the tower is considered as a beam-like structure clamped at ground level, the first mode represents the first bending mode around the weak bending axis (Y-axis in Fig. 9), while the second mode represents the first bending mode around the strong bending axis (X-axis in Fig. 9). The third mode is a torsion mode. Modes four, five and six represent the second bending mode around the weak axis, the second bending mode around the strong axis, and the second torsion mode, respectively.

Since the identified state-space model allows for general viscous damping, the identified mode shapes are complex vectors [32]. However, when soil-structure interaction effects are negligible, real normal modes are expected because localized dampers are not present and the mode shapes are regular standing waves ${ }^{1}$. Fig. 10 shows the modal phase collinearity (MPC) values for the identified mode shapes. For a given mode shape, the MPC is a positive real quantity that takes values between zero and unity [34]. Values close to unity indicate perfect mode shape collinearity, while significantly lower values indicate strongly complex mode shapes. It can be seen that the MPC of the first three modes is larger than 0.95, while for modes four to six the values are $0.68,0.59$, and 0.41 , respectively. Since real normal modes are expected, this suggests that the first three modes are identified with high quality, while estimates of modal characteristics of the last three modes are of much lower quality.

\subsection{Uncertainty quantification of the estimated modal characteristics}

The estimates of the eigenfrequencies and damping ratios, obtained for each of the ten different setups, are shown in Fig. 11 and Fig. 12, respectively, together with their $95 \%$ confidence intervals. The accuracy of the eigenfrequency estimates of the first three modes, measured by their predicted $95 \%$ confidence interval, lies between $0.002 \mathrm{~Hz}$ and $0.008 \mathrm{~Hz}$, while for the last three modes it lies between $0.02 \mathrm{~Hz}$ and $0.31 \mathrm{~Hz}$. For the damping ratios, the accuracy of the estimates of the first three modes lies between $0.3 \%$ and $0.8 \%$, while for the last three modes this lies between $0.9 \%$ and $10.1 \%$. These results therefore confirm quantitatively that the estimates of modes 1,2 and 3 are much more accurate than the estimates of modes 4, 5 and 6. This is probably due to the fact that these modes are less well excited by the ambient wind loading, and to a non-perfect synchronization between the wireless units. Note also that the accuracy of the estimated values may vary considerably from setup to setup.

Table 3: IMEC Tower: eigenfrequencies (in Hz): sample mean and sample standard deviation of the point estimates and sample mean and sample standard deviation of the standard deviation estimates, computed over the ten setups.

\begin{tabular}{llrrrrrr}
\hline \multicolumn{1}{l}{ Mode no. } & & 1 & 2 & 3 & 4 & 5 & 6 \\
\hline$\mu_{\text {samp }}\left(\hat{f}_{k}\right)$ & {$[\mathrm{Hz}]$} & 0.5529 & 0.7519 & 1.2177 & 2.7385 & 3.2777 & 3.9383 \\
$\sigma_{\text {samp }}\left(\hat{f}_{k}\right)$ & {$[\mathrm{Hz}]$} & 0.0012 & 0.0008 & 0.0014 & 0.0365 & 0.0100 & 0.0336 \\
$\mu_{\text {samp }}\left(\hat{\sigma}\left(\hat{f}_{k}\right)\right)[\mathrm{Hz}]$ & 0.0008 & 0.0008 & 0.0013 & 0.0222 & 0.0129 & 0.0338 \\
$\sigma_{\text {samp }}\left(\hat{\sigma}\left(\hat{f}_{k}\right)\right)[\mathrm{Hz}]$ & 0.0001 & 0.0002 & 0.0003 & 0.0097 & 0.0070 & 0.0239 \\
\hline$\mu_{\text {samp }}\left(\hat{\xi}_{k}\right)$ & {$[\%]$} & 0.68 & 0.58 & 0.67 & 6.10 & 2.94 & 4.10 \\
$\sigma_{\text {samp }}\left(\hat{\xi}_{k}\right)$ & {$[\%]$} & 0.13 & 0.11 & 0.11 & 0.81 & 0.51 & 1.22 \\
$\mu_{\text {samp }}\left(\hat{\sigma}\left(\hat{\xi}_{k}\right)\right)[\%]$ & 0.14 & 0.11 & 0.09 & 0.79 & 0.34 & 0.98 \\
$\sigma_{\text {samp }}\left(\hat{\sigma}\left(\hat{\xi}_{k}\right)\right)[\%]$ & 0.04 & 0.02 & 0.02 & 0.40 & 0.12 & 0.64 \\
\hline
\end{tabular}

Table 3 compares the sample standard deviation of the eigenfrequency and damping ratio estimates, obtained over the ten different setups, with the standard deviation estimates, computed in each setup. The tabulated quantities have

\footnotetext{
${ }^{1}$ Note that in structures with multiple symmetries, such as axisymmetric shell structures, mode shapes may take the form of running waves [33].
} 

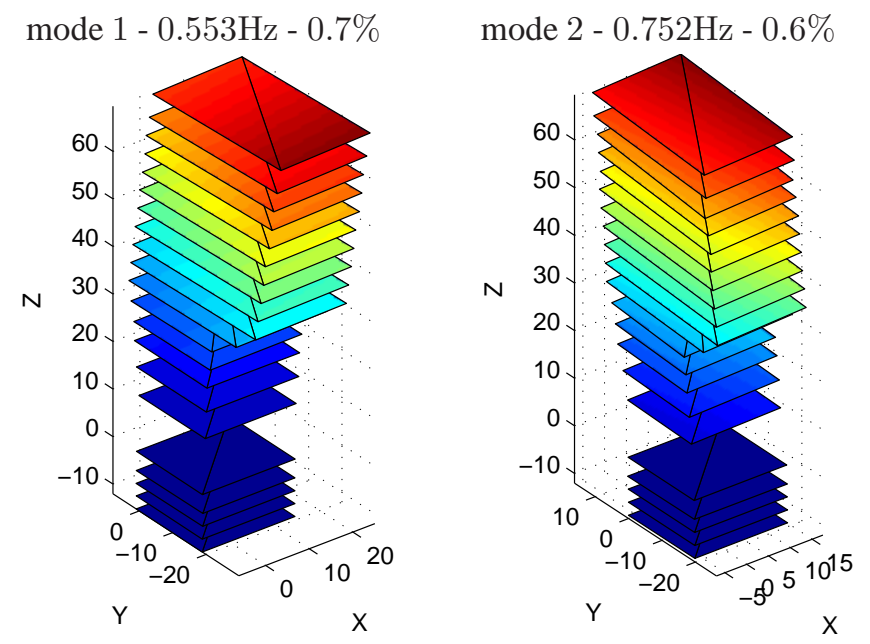

mode $3-1.218 \mathrm{~Hz}-0.7 \%$
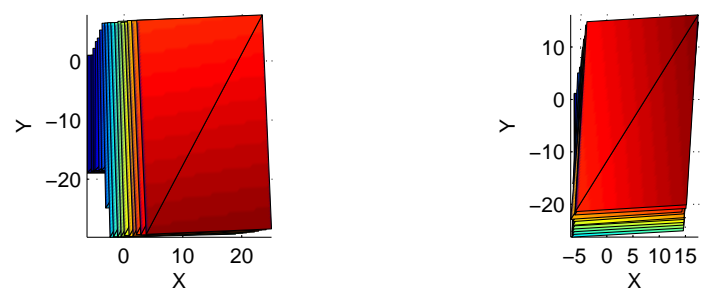

mode $4-2.743 \mathrm{~Hz}-6.2 \%$

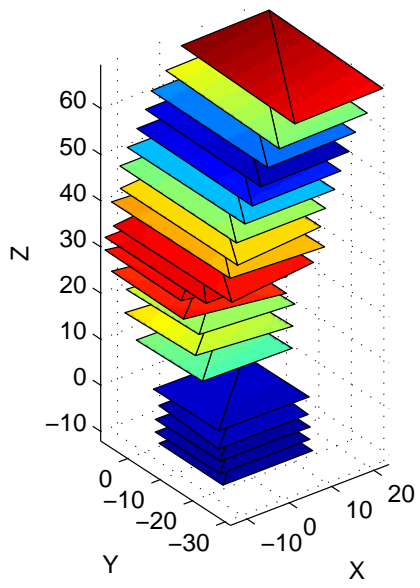

mode $5-3.278 \mathrm{~Hz}-2.9 \%$
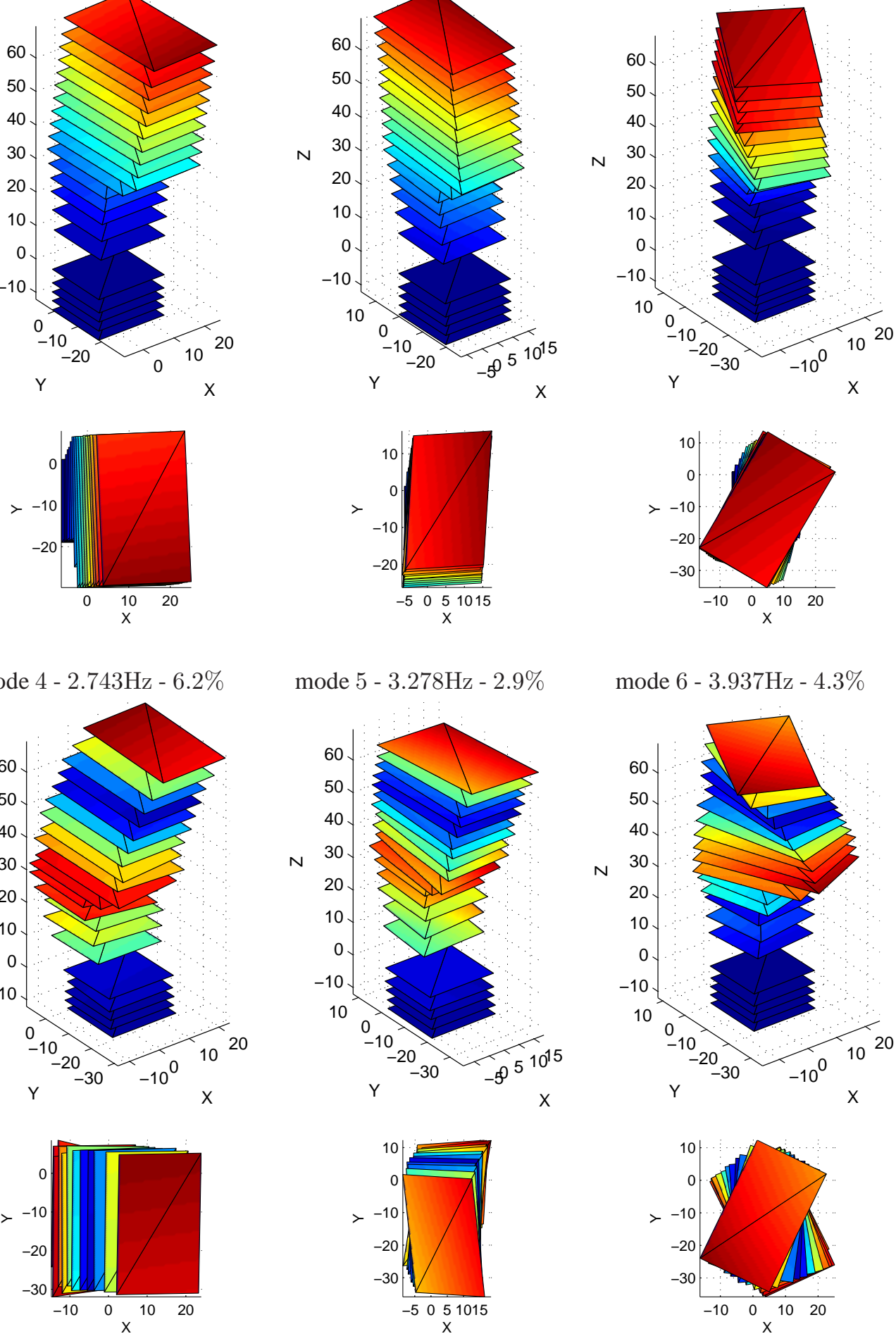

mode $6-3.937 \mathrm{~Hz}-4.3 \%$

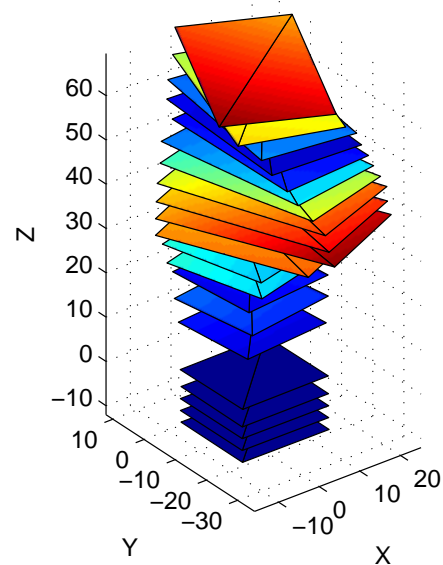

Figure 9: IMEC Tower: identified eigenfrequencies, damping ratios and displacement mode shapes. 


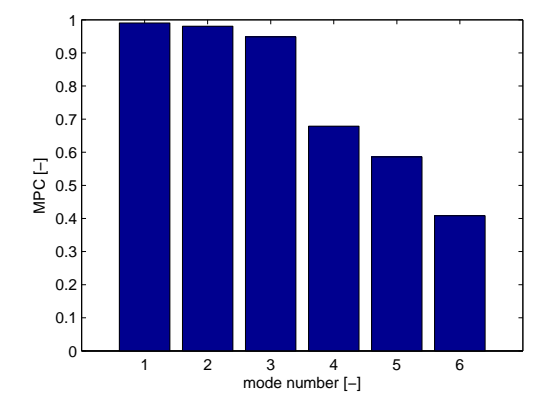

Figure 10: IMEC Tower: modal phase collinearity values of the identified mode shapes.
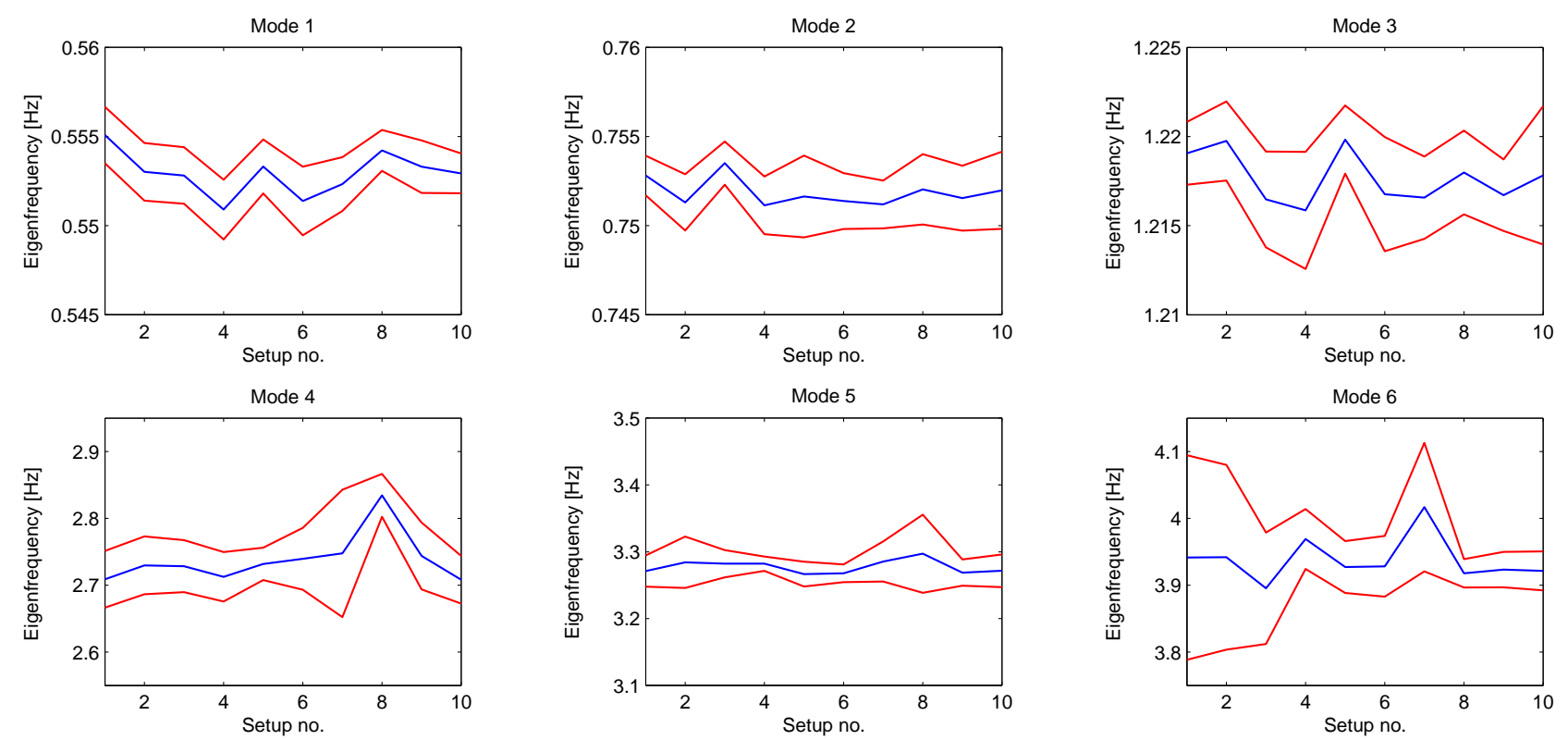

Figure 11: IMEC Tower: estimated eigenfrequencies and $95 \%$ confidence intervals for all setups. Note the different scale of the vertical axis for the first three and last three modes.

been defined previously, in section 3.3. The observed (or sample) standard deviation and the estimated standard deviation should agree well when the number of setups is large, the ambient excitation is stationary and the measurement setup is equally informative amongst all setups. Although this is not entirely the case here, a reasonably good agreement is still expected since there was no pronounced change in wind conditions during the measurement period and since the reference outputs are the same for all setups. Considering the variability of the estimated standard deviations across the different setups and the limited number of setups, the sample standard deviations agree well with the estimated standard deviations.

\section{Conclusions}

A method for estimating the variance of modal characteristics that are identified with stochastic subspace identification has been validated by application to vibration data gathered on a prestressed concrete bridge and a mid-rise building. For each of both structures, two validations were performed. A first validation consisted of comparing the predicted uncertainty with the observed variability of the eigenfrequencies and damping ratios between the different setups. The variances that are predicted by the method for each setup were found in good agreement with the sample variances 

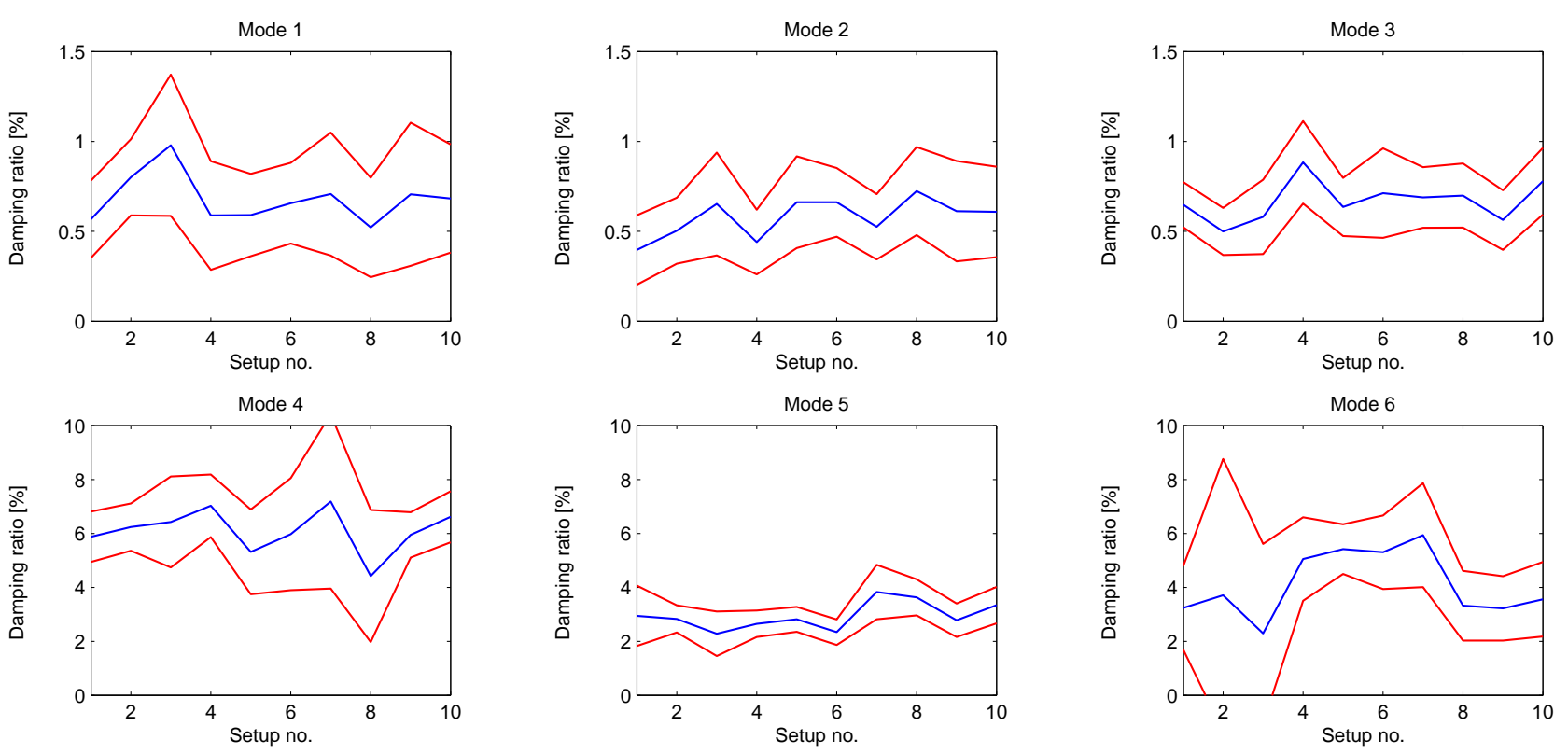

Figure 12: IMEC Tower: estimated damping ratios and $95 \%$ confidence intervals for all setups. Note the different scale of the vertical axis for the first three and last three modes.

computed over all setups, especially when the estimation accuracy was similar in the different setups. A second validation focused on the differences in estimation accuracy between different modes and different setups. Also in this case, the uncertainty of the estimates as predicted by the method was consistent with the experimental observations: estimates that were known to be less reliably, e.g. because they related to non-smooth mode shapes, consistently showed a larger predicted variance.

\section{Acknowledgements}

This research was supported by the Research Foundation - Flanders (FWO), Belgium, by means of a Postdoctoral Research Fellowship provided to the first author and through Research Project G099014N. The authors are all members of KU Leuven BOF PFV/10/002 Optimization in Engineering Center (OPTEC). They would like to thank the colleagues from the Ministry of Equipment and Transport of the Walloon Region, the Besix construction company and the Department of Civil Engineering of KU Leuven who helped in collecting the vibration data for this study.

\section{Appendix: Derivatives of modal characteristics with respect to state-space matrices}

Equations (6) and (7) contain the relation between the eigenfrequency $f_{k}$ and damping ratio $\xi_{k}$ (in $\%$ of critical) of mode $k$, and the matrix $\mathbf{A}$ of the state-space model. A linear sensitivity analysis of this relationship has been performed in [1]. The result reads

$$
\Delta\left[\begin{array}{l}
f_{k} \\
\xi_{k}
\end{array}\right]=\mathbf{J}_{\mathrm{f} \xi, k} \operatorname{vec}(\Delta \mathbf{A})
$$

with

$$
\mathbf{J}_{\mathrm{f} \xi, k}=\frac{1}{T\left|\lambda_{k}\right|^{2}\left|\lambda_{\mathrm{ck}}\right|} \operatorname{re}\left(\frac{\left(\boldsymbol{\psi}_{k} \otimes \overline{\chi_{k}}\right)^{\mathrm{T}}}{\chi_{k}^{\mathrm{H}} \boldsymbol{\psi}_{k}}\right)\left[\begin{array}{cc}
\mathcal{R}\left(\lambda_{k}\right) & \mathcal{I}\left(\lambda_{k}\right) \\
-\mathcal{I}\left(\lambda_{k}\right) & \mathcal{R}\left(\lambda_{k}\right)
\end{array}\right]\left[\begin{array}{cc}
\frac{\mathcal{R}\left(\lambda_{c k}\right)}{2 \pi} & \frac{\mathcal{I}\left(\lambda_{\mathrm{ck}}\right)}{2 \pi} \\
-100+\frac{\mathcal{R}\left(\lambda_{c k}\right)^{2}}{\left|\lambda_{c k}\right|^{2}} & 100 \frac{\mathcal{R}\left(\lambda_{\mathrm{c} k}\right) \mathcal{I}\left(\lambda_{\mathrm{c} k}\right)}{\left|\lambda_{c k}\right|^{2}}
\end{array}\right]
$$


where re () stacks the real part of a matrix on top of the imaginary part, the overbar denotes complex conjugate, the superscript $H$ denotes complex conjugate (or Hermitian) transpose, the Kronecker product is denoted with $\otimes$, and $\chi_{k}$ denotes the left eigenvector of matrix $\mathbf{A}$, corresponding to the eigenvalue $\lambda_{k}$ and right eigenvector $\boldsymbol{\psi}_{k}$ :

$$
\boldsymbol{A}^{\mathrm{H}} \chi_{k}=\bar{\lambda}_{k} \chi_{k} \quad \text { with, as before, } \quad \boldsymbol{A} \psi_{k}=\lambda_{k} \psi_{k} .
$$

Equations (6) and (8) contain the relationship between the unscaled mode shape $\phi_{k}$ of mode $k$, and the matrices $\mathbf{A}$ and $\mathbf{C}$ of the state-space model. A linear sensitivity analysis of this relationship has also been performed in [1]. The result reads

$$
\Delta \phi_{k}=\mathbf{J}_{\phi, k}\left[\begin{array}{c}
\operatorname{vec}(\Delta \boldsymbol{A}) \\
\operatorname{vec}(\Delta \boldsymbol{C})
\end{array}\right]
$$

with

$$
\mathbf{J}_{\phi, k}=\left[\left(\mathbf{I}-\frac{\mathbf{A}}{\lambda_{k}}\right)^{\dagger} \frac{1}{\lambda_{k}}\left(\boldsymbol{\psi}_{k}^{\mathrm{T}} \otimes\left(\mathbf{I}-\frac{\boldsymbol{\psi}_{k} \boldsymbol{\chi}_{k}^{\mathrm{H}}}{\boldsymbol{\chi}_{k}^{\mathrm{H}} \boldsymbol{\psi}_{k}}\right)\right) \quad \boldsymbol{\psi}_{k}^{\mathrm{T}} \otimes \mathbf{I}_{n_{\mathrm{y}}}\right],
$$

where $\dagger$ denotes the pseudo-inverse.

From these expressions, the Jacobian $\mathbf{J}_{k}$ which appears in the variance equation (22) for the estimated eigenfrequency $\hat{f}_{k}$, damping ratio $\hat{\xi}_{k}$ and mode shape $\hat{\phi}_{k}$ is obtained as

$$
\mathbf{J}_{k}=\left[\begin{array}{c}
{\left[\begin{array}{ll}
\mathbf{J}_{\mathrm{f} \xi, k} & \mathbf{0}
\end{array}\right]} \\
\operatorname{re}\left(\mathbf{J}_{\phi, k}\right)
\end{array}\right] .
$$

The mode shapes $\hat{\phi}_{k}$ that result from the SSI method are often normalized (or re-scaled). In [1], the unit modal displacement normalization scheme is considered where, in each possible realization of the stochastic ensemble, a single element $\hat{\phi}_{k, l}$ of the mode shape, equals unity:

$$
\hat{\varphi}_{k}=\frac{\hat{\phi}_{k}}{\hat{\phi}_{k, l}} .
$$

In this scheme, the variance of the real and imaginary parts of $\hat{\varphi}_{k, l}$ equals zero because $\hat{\varphi}_{k, l}=1$ is a deterministic quantity. A consequence of this scheme is therefore that the mode shape uncertainty is unequally spread amongst the different degrees of freedom.

An alternative normalization scheme is also considered here. It consists of two normalization steps: (1) rotating $\hat{\phi}_{k}$ such that one of its components, $\hat{\phi}_{k, l}$, has unit norm, and (2) re-scaling $\hat{\phi}_{k}$ such that its Euclidian norm has unit length. Application of the first step yields

$$
\hat{\varphi}_{1, k}:=\hat{\phi}_{k} \mathrm{e}^{-i \alpha_{k}, l},
$$

where $\alpha_{k, l}$ is the phase angle of $\hat{\phi}_{k, l}$ :

$$
\hat{\phi}_{k, l}=t_{k, l} \mathrm{e}^{i \alpha_{k, l}} .
$$

This first step has already been considered in [20], where the corresponding Jacobian was also derived:

$$
\mathbf{J}_{\varphi 1, k}=\operatorname{re}\left(\mathrm{e}^{-i \alpha_{k, l}}\left(-i t_{k, l}^{-2} \hat{\boldsymbol{\phi}}_{k}\left[-\mathcal{I}\left(\hat{\phi}_{k, l}\right) \mathbf{e}_{k}^{\mathrm{T}} \quad \mathcal{R}\left(\hat{\phi}_{k, l}\right) \mathbf{e}_{k}^{\mathrm{T}}\right]+\left[\begin{array}{ll}
1 & i
\end{array}\right] \otimes \mathbf{I}_{n_{\mathrm{y}}}\right)\right) .
$$

Application of the second step results in

$$
\hat{\varphi}_{2, k}:=\frac{\hat{\varphi}_{1, k}}{\left\|\hat{\varphi}_{1, k}\right\|} .
$$

This step second step has not been considered previously. A first-order sensitivity analysis results in

$$
\begin{aligned}
\Delta \hat{\boldsymbol{\varphi}}_{2, k} & =\frac{1}{\left\|\hat{\boldsymbol{\varphi}}_{1, k}\right\|} \Delta \hat{\boldsymbol{\varphi}}_{1, k}-\frac{1}{2} \hat{\boldsymbol{\varphi}}_{1, k}\left\|\hat{\boldsymbol{\varphi}}_{1, k}\right\|^{-3} \Delta\left\|\hat{\boldsymbol{\varphi}}_{1, k}\right\| \\
& =\left(\frac{1}{\left\|\hat{\boldsymbol{\varphi}}_{1, k}\right\|}-\hat{\boldsymbol{\varphi}}_{1, k}\left\|\hat{\boldsymbol{\varphi}}_{1, k}\right\|^{-3} \operatorname{re}\left(\hat{\boldsymbol{\varphi}}_{1, k}\right)^{\mathrm{T}}\right) \Delta \operatorname{re}\left(\hat{\boldsymbol{\varphi}}_{1, k}\right),
\end{aligned}
$$


so the corresponding Jacobian reads

$$
\mathbf{J}_{\varphi 2, k}=\frac{\mathbf{I}_{2 n_{\mathrm{y}}}}{\left\|\hat{\boldsymbol{\varphi}}_{1, k}\right\|}-\operatorname{re}\left(\hat{\boldsymbol{\varphi}}_{1, k}\right)\left\|\hat{\boldsymbol{\varphi}}_{1, k}\right\|^{-3} \operatorname{re}\left(\hat{\boldsymbol{\varphi}}_{1, k}\right)^{\mathrm{T}} .
$$

Combining both results, one has that

$$
\operatorname{re}\left(\hat{\boldsymbol{\varphi}}_{2, k}\right)=\mathbf{J}_{\varphi 2, k} \mathbf{J}_{\varphi 1, k} \mathrm{re}\left(\hat{\phi}_{k}\right)
$$

\section{References}

[1] E. Reynders, R. Pintelon, G. De Roeck,Uncertainty bounds on modal parameters obtained from Stochastic Subspace Identification, Mechanical Systems and Signal Processing 22 (4) (2008) 948-969.

[2] R. Pintelon, P. Guillaume, J. Schoukens, Uncertainty calculation in (operational) modal analysis, Mechanical Systems and Signal Processing 21 (6) (2007) 2359-2373.

[3] B. Peeters, G. De Roeck, Stochastic system identification for operational modal analysis: A review, ASME Journal of Dynamic Systems, Measurement, and Control 123 (4) (2001) 659-667.

[4] F. Magalhaẽs, A. Cunha, Explaining operational modal analysis with data from an arch bridge, Mechanical Systems and Signal Processing 25 (5) (2011) 1431-1450.

[5] E. Reynders, System identification methods for (operational) modal analysis: review and comparison, Archives of Computational Methods in Engineering 19 (1) (2012) 51-124.

[6] M. Aoki, State space modelling of time series, Springer-Verlag, Berlin, Germany, 1987.

[7] B. Peeters, G. De Roeck, Reference-based stochastic subspace identification for output-only modal analysis, Mechanical Systems and Signal Processing 13 (6) (1999) 855-878.

[8] J.-N. Juang, R. Pappa, An eigensystem realization algorithm for modal parameter identification and model reduction, Journal of Guidance, Control and Dynamics 8 (5) (1985) 620-627.

[9] G. James, T. Carne, J. Lauffer, The natural excitation technique (NExT) for modal parameter extraction from operating structures, Modal Analysis: the International Journal of Analytical and Experimental Modal Analysis 10 (4) (1995) 260-277.

[10] T. Carne, G. James III, The inception of OMA in the development of modal testing technology for wind turbines, Mechanical Systems and Signal Processing 24 (5) (2010) 1213-1226.

[11] P. Van Overschee, B. De Moor, Subspace identification for linear systems, Kluwer Academic Publishers, Dordrecht, The Netherlands, 1996.

[12] D. Bauer, Comparing the CCA subspace method to pseudo maximum likelihood methods in the case of no exogenous inputs, Journal of Time Series Analysis 26 (5) (2005) 631-668

[13] L. Ljung, System identification: theory for the user, 2nd Edition, Prentice Hall, Upper Saddle River, NJ, 1999.

[14] R. Pintelon, J. Schoukens, System Identification, IEEE Press, New York, NY, 2001.

[15] R. Pintelon, J. Schoukens, P. Guillaume, Box-Jenkins identification revisited - part III: Multivariable systems, Automatica 43 (5) (2006) 868-875.

[16] P. Guillaume, L. Hermans, H. Van der Auweraer, Maximum likelihood identification of modal parameters from operational data, in: Proceedings of the 17th International Modal Analysis Conference, Kissimmee, FL, 1999, pp. 1887-1893.

[17] S.-K. Au, Fast Bayesian ambient modal identification in the frequency domain, part I: posterior most probable value, Mechanical Systems and Signal Processing 26 (2012) 60-75.

[18] T. De Troyer, P. Guillaume, R. Pintelon, S. Vanlanduit, Fast calculation of confidence intervals on parameter estimates of least-squares frequencydomain estimators, Mechanical Systems and Signal Processing 23 (2) (2009) 261-273.

[19] M. Döhler, L. Mevel, Efficient multi-order uncertainty computation for stochastic subspace identification, Mechanical Systems and Signal Processing 38 (2) (2013) 346-366.

[20] M. Döhler, X.-B. Lam, , L. Mevel, Uncertainty quantification for modal parameters from stochastic subspace identification on multi-setup measurements, Mechanical Systems and Signal Processing 36 (2) (2013) 562-581.

[21] K. Arun, S. Kung, Balanced approximation of stochastic systems, SIAM journal on matrix analysis and applications 11 (1990) $42-68$.

[22] E. Jaynes, Probability Theory. The Logic of Science, Cambridge University Press, Cambridge, UK, 2003.

[23] H. Zeiger, A. McEwen, Approximate linear realizations of given dimension via Ho's algorithm, IEEE Transactions on Automatic Control 19 (1974) 153.

[24] S. Kung, A new identification and model reduction algorithm via singular value decomposition, in: Proceedings of the 12th Asilomar conference on circuits, systems and computers, Pacific Grove, CA, 1978, pp. 705-714.

[25] E. Reynders, J. Houbrechts, G. De Roeck, Fully automated (operational) modal analysis, Mechanical Systems and Signal Processing 29 (2012) $228-250$.

[26] E. Reynders, G. De Roeck, Reference-based combined deterministic-stochastic subspace identification for experimental and operational modal analysis, Mechanical Systems and Signal Processing 22 (3) (2008) 617-637.

[27] E. Carden, A. Mita, Challenges in developing confidence intervals on modal parameters estimated for large civil infrastructure with stochastic subspace identification, Structural Control and Health Monitoring 18 (1) (2011) 53-78.

[28] E. Reynders, G. De Roeck, P. Bakir, C. Sauvage, Damage identification on the Tilff bridge by vibration monitoring using optical fibre strain sensors, ASCE Journal of Engineering Mechanics 133 (2) (2007) 185-193.

[29] A. Pandey, M. Biswas, M. Samman, Damage detection from changes in curvature mode shapes, Journal of Sound and Vibration 145 (2) (1991) $321-332$.

[30] E. Reynders, G. Wursten, G. De Roeck, Output-only structural health monitoring in changing environmental conditions by means of nonlinear system identification, Structural Health Monitoring 13 (1) (2014) 82-93. 
[31] F. Magalhaẽs, A. Cunha, E. Caetano, Online automatic identification of the modal parameters of a long span arch bridge, Mechanical Systems and Signal Processing 23 (2) (2009) 316-329.

[32] D. J. Ewins, Modal testing, 2nd Edition, Research Studies Press, Baldock, U.K., 2000.

[33] D. Dooms, G. Degrande, G. De Roeck, E. Reynders, Finite element modelling of a silo based on experimental modal analysis, Engineering Structures 28 (4) (2006) 532-542.

[34] R. S. Pappa, K. B. Elliott, A. Schenk, A consistent-mode indicator for the eigensystem realization algorithm, Report NASA TM-107607, National Aeronautics and Space Administration (April 1992). 